\title{
Noetherianity up to symmetry
}

\author{
Jan Draisma \\ Department of Mathematics and Computer Science, Eindhoven Uni- \\ versity of Technology; and Centrum Wiskunde en Informatica, Am- \\ STERDAM; THE Netherlands j.draisma@tue.nl
}


Abstract. These lecture notes for the 2013 CIME/CIRM summer school Combinatorial Algebraic Geometry deal with manifestly infinite-dimensional algebraic varieties with large symmetry groups. So large, in fact, that subvarieties stable under those symmetry groups are defined by finitely many orbits of equations - whence the title Noetherianity up to symmetry. It is not the purpose of these notes to give a systematic, exhaustive treatment of such varieties, but rather to discuss a few "personal favourites": exciting examples drawn from applications in algebraic statistics and multilinear algebra. My hope is that these notes will attract other mathematicians to this vibrant area at the crossroads of combinatorics, commutative algebra, algebraic geometry, statistics, and other applications.

Acknowledgments The author was supported by a Vidi grant from the Netherlands Organisation for Scientific Research (NWO).

Eindhoven, Fall 2013

Jan Draisma 


\section{Contents}

Chapter 1. Kruskal's Tree Theorem 5

Chapter 2. Equivariant Gröbner bases 9

Chapter 3. Equivariant Noetherianity

Chapter 4. Chains of varieties

Chapter 5. The independent set theorem 23

Chapter 6. The Gaussian $k$-factor model 27

Chapter 7. Tensors and $\Delta$-varieties $\quad 29$

Bibliography 37 



\section{CHAPTER 1}

\section{Kruskal's Tree Theorem}

All finiteness proofs in these lecture notes are based on a beautiful combinatorial theorem due to Kruskal. In fact, the special case of that theorem known as Higman's Lemma suffices for all of those proofs. But, hoping that Kruskal's Tree Theorem will soon find further applications in infinite-dimensional algebraic geometry, I have decided to prove the theorem in its full strength. Original sources for Kruskal's Tree Theorem and Higman's Lemma are Kru60 and Hig52, respectively. We follow closely the beautiful proof in NW63. Throughout we use the notation $\mathbb{N}:=\{1,2, \ldots\}, \mathbb{Z}_{\geq 0}:=\{0,1, \ldots\}$, and $[n]:=\{1, \ldots, n\}$ for $n \in \mathbb{Z}_{\geq 0}$. In particular, we have $[0]=\emptyset$.

The main concept is that of a well-partial-order on a set $S$. This is a partial order $\leq$ with the property that for any infinite sequence $s_{1}, s_{2}, \ldots$ of elements of $S$ there exists a pair of indices $i<j$ with $s_{i} \leq s_{j}$. Arguing by contradiction one then proves that there exists an index $i$ such that $s_{j} \geq s_{i}$ holds for infinitely many indices $j>i$. Take the first such index $i_{1}$, and retain only the term $s_{i_{1}}$ together with the infinitely many terms $s_{j}$ with $j>i_{1}$ and $s_{j} \geq s_{i_{1}}$. Among these pick an index $i_{2}>i_{1}$ in a similar fashion, etc. This leads to the conclusion that in a wellpartially-ordered set any infinite sequence has an infinite ascending subsequence $s_{i_{1}} \leq s_{i_{2}} \leq \ldots$ with $i_{1}<i_{2}<\ldots$

Examples of well-partial-orders are partial orders on finite sets, and well-orders (which are linear well-partial-orders). If two sets $S, T$ are both equipped with wellpartial-orders, then the componentwise partial order on the Cartesian product $S \times T$ defined by $(s, t) \leq\left(s^{\prime}, t^{\prime}\right)$ if and only if $s \leq s^{\prime}$ and $t \leq t^{\prime}$ is again a well-partial-order. Indeed, in an infinite sequence $\left(s_{1}, t_{1}\right),\left(s_{2}, t_{2}\right), \ldots$ there is an infinite subsequence of indices where the $s_{i}$ increase weakly and in that subsequence there exist a pair of indices $i \leq j$ where in addition to $s_{i} \leq s_{j}$ also the inequality $t_{i} \leq t_{j}$ holds.

Repeatedly applying this Cartesian-product construction with all factors equal to the non-negative integers $\mathbb{Z}_{\geq 0}$ one obtains the statement that the componentwise order on $\mathbb{Z}_{\geq 0}^{n}$ is a well-partial-order. This fact, known as Dickson's Lemma, can be used to prove Hilbert's Basis Theorem. In a similar fashion we shall use Kruskal's Tree Theorem to prove Noetherianity of certain rings up to symmetry. Before stating and proving Kruskal's Tree Theorem, we first discuss the following special case.

Lemma 1.1. For any well-partial-order on a set $S$ the partial order on the set of finite multi-subsets of $S$ defined by $A \leq B$ if and only if there exists an injective map $f: A \rightarrow B$ with $a \leq f(a)$ for all $a \in A$ is a well-partial-order.

Proof. Suppose that it is not. Then there exists an infinite sequence $A_{1}, A_{2}, \ldots$ of finite multi-subsets of $S$ such that $A_{i} \not \leq A_{j}$ for all pairs of indices $i<j$. Such a 

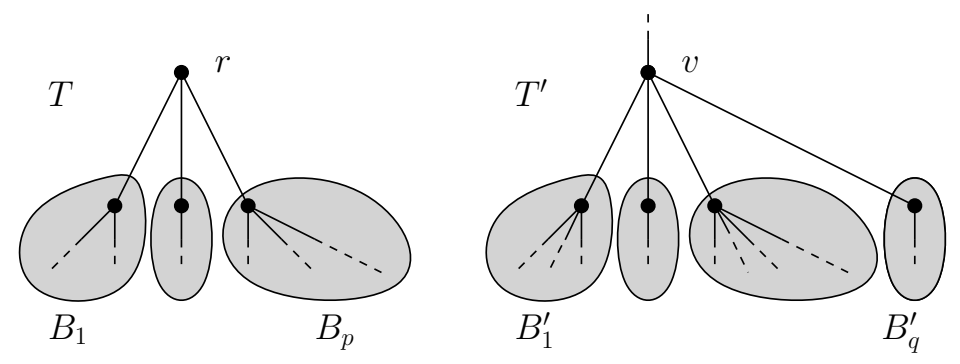

Figure 1.1. If the $S$-label of $r$ is at most that of $v$, and if $B_{i} \leq$ $B_{\pi(i)}^{\prime}$ for some injective $\pi:[p] \rightarrow[q]$, then $T \leq T^{\prime}$.

sequence is called a bad sequence, and we may assume that it is minimal in the following sense. First, the cardinality $\left|A_{1}\right|$ of $A_{1}$ is minimal among all bad sequences. Second, $\left|A_{2}\right|$ is minimal among all bad sequences starting with $A_{1}$, etc.

As the empty multi-set is smaller than all other multi-sets, none of the multisets $A_{i}$ is empty, so we may choose an element $a_{i}$ from each $A_{i}$ and define $B_{i}:=$ $A_{i} \backslash\left\{a_{i}\right\}$. There exists an infinite subsequence $i_{1}<i_{2}<\ldots$ where $a_{i_{1}} \leq a_{i_{2}} \leq \ldots$. Now the desired contradiction will follow by considering the sequence

$$
A_{1}, A_{2}, \ldots, A_{i_{1}-1}, B_{i_{1}}, B_{i_{2}}, \ldots
$$

Indeed, no $A_{i}$ with $i \leq i_{1}-1$ is less than or equal to $A_{j}$ with $i<j \leq i_{1}-1$. But neither is any $A_{i}$ with $i \leq i_{1}-1$ less than or equal to any $B_{j}$ with $j \in\left\{i_{1}, i_{2}, \ldots\right\}$, or else we would have $A_{i} \leq B_{j} \leq A_{j}$, with the inclusion map witnessing the second inequality. Finally, no relation $B_{i} \leq B_{j}$ holds with $i, j \in\left\{i_{1}, i_{2}, \ldots\right\}$ and $i<j$. Indeed, a map $B_{i} \rightarrow B_{j}$ witnessing that inequality could be extended to a map $A_{i} \rightarrow A_{j}$ witnessing $A_{i} \leq A_{j}$ by mapping $a_{i}$ to $a_{j}$. We conclude that the new sequence is bad, but this contradicts the minimality of $\left|A_{i_{1}}\right|$ among all bad sequences starting with $A_{1}, \ldots, A_{i_{1}-1}$.

The general case of Kruskal's Tree Theorem concerns the set of (isomorphism classes of) finite, rooted trees whose vertices are labelled with elements of a fixed partially ordered set $S$. We call such objects $S$-labelled trees. A partial order on $S$-labelled trees is defined recursively as follows; see also Figure 1.1. Suppose that $T$ is an $S$-labelled tree with root $r$ and suppose that $T$ branches at $r$ into trees $B_{1}, \ldots, B_{p}$ whose roots are the children of $r ; p=0$ is allowed here, and renders void one of the conditions that follow. We say that $T$ is less than or equal to a second $S$-labelled tree $T^{\prime}$ if the latter has a vertex $v$ (not necessarily its root) where $T^{\prime}$ branches into trees $B_{1}^{\prime}, \ldots, B_{q}^{\prime}$ (rooted at children of $v$ ), such that the $S$-label of $v$ is at least that of $r$ and such that there exists an injective map $\pi$ from [p] into $[q]$ with $B_{i} \leq B_{\pi(i)}^{\prime}$ for all $i$. Unfolding this recursive definition one finds that the one-dimensional CW-complex given by the tree $T$ can then be homeomorphically embedded into that of $T^{\prime}$ in such a way that each vertex $u$ of $T$ gets mapped into a vertex of $T^{\prime}$ whose $S$-label is at least that of $u$; see Figure 1.2 .

TheOREm 1.2 (Kruskal's Tree Theorem). For any non-empty well-partiallyordered set $S$, the set of $S$-labelled trees is well-partially-ordered by the partial order just defined. 


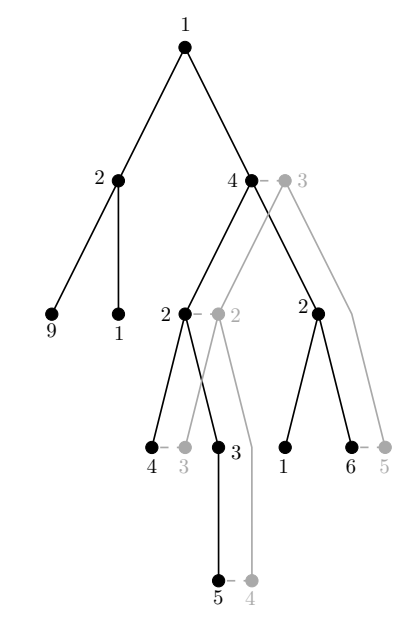

Figure 1.2. The $\mathbb{N}$-labelled tree in gray is smaller than the $\mathbb{N}$ labelled tree in black in Kruskal's order.

Note that the lemma is, indeed, a special case of this theorem, obtained by restricting to star trees with a root (labelled with some fixed, irrelevant element of $S)$ connected directly to all its leaves.

Proof. The proof is very similar to that of the lemma. Assume, for a contradiction, the existence of a bad sequence $T_{1}, T_{2}, \ldots$, which we may take minimal in the sense that the cardinality of the vertex set of $T_{i}$ is minimal among all bad sequences starting with $T_{1}, \ldots, T_{i-1}$. At its root, $T_{i}$ branches into a finite multi-set $R_{i}$ of smaller $S$-labelled trees. Let $R$ be the set-union of all $R_{i}$ as $i$ runs through $\mathbb{N}$, so that $R$ is a set of $S$-labelled trees. If $R$ contained a bad sequence, then it would contain a bad sequence $B_{i_{1}}, B_{i_{2}}, \ldots$ with $B_{i_{j}} \in R_{i_{j}}$ and $i_{1}<i_{2}<\ldots$ Then, as in the proof of the lemma, one shows that the sequence $T_{1}, \ldots, T_{i_{1}-1}, B_{i_{1}}, B_{i_{2}}, \ldots$ would be a bad sequence of trees contradicting the minimality of the original sequence. Hence $R$ is well-partially-ordered.

Consider a subsequence $T_{k_{1}}, T_{k_{2}}, \ldots$ with $k_{1}<k_{2}<\ldots$ for which the root labels of the $T_{k_{i}}$ weakly increase in $S$. Applying the lemma to the well-partiallyordered set $R$, we find that there exist $j<l$ such that $R_{k_{j}} \leq R_{k_{l}}$. But then $T_{k_{j}} \leq T_{k_{l}}$ (by mapping the root of $T_{k_{j}}$ to the root of $T_{k_{l}}$ and $R_{k_{j}}$ suitably into $R_{k_{l}}$ ), a contradiction.

We now formulate Higman's Lemma, which is a useful direct consequence of Kruskal's Tree Theorem. Given a partially ordered set $S$, define a partial order on the set $S^{*}=\bigcup_{p} S^{p}$ of finite sequences over $S$ as follows. A sequence $\left(s_{1}, \ldots, s_{p}\right)$ is less than or equal to a sequence $\left(s_{1}^{\prime}, \ldots, s_{q}^{\prime}\right)$ if there exists a strictly increasing map $\pi:[p] \rightarrow[q]$ satisfying $s_{i} \leq s_{\pi(i)}^{\prime}$ for all $i$.

THEOREM 1.3 (Higman's Lemma). For any well-partially-ordered set $S$, the partial order on $S^{*}$ just defined is a well-partial-order.

Proof. Encode a sequence $\left(s_{1}, \ldots, s_{p}\right)$ in $S^{*}$ as an $S$-labelled tree with root 1 labelled $s_{1}$, with a single child 2 labelled $s_{2}$, etc. Under this encoding the partial 
order on $S^{*}$ agrees with that on $S$-labelled trees, so that Kruskal's theorem implies Higman's Lemma. 


\section{CHAPTER 2}

\section{Equivariant Gröbner bases}

Just like, through a leading term argument, Dickson's Lemma implies Hilbert's Basis Theorem, Higman's Lemma implies the central finiteness result that all our later proofs build upon. What follows is certainly not the most general setting, but it will suffice our purposes. For much more on this theme see Coh67, Coh87, AH07, AH08, DS06, HS12, HMdC13, LSL09.

Let $X$ be a (typically infinite) set of variables, and let Mon denote the free commutative monoid of monomials in those variables. Let $\leq$ be a monomial order on Mon, i.e., a well-order that satisfies the additional condition that $u \leq v \Rightarrow u w \leq$ $v w$ for all $u, v, w \in$ Mon. Let $\Pi$ be a (typically non-commutative) monoid acting from the left on Mon by means of monoid endomorphisms and assume that the action preserves strict inequalities, i.e., $u<v$ implies $\pi u<\pi v$ for all $\pi \in \Pi$. In particular, $\pi$ acts by means of an injective map on Mon. Moreover, we have $\pi u \geq u$ since otherwise the sequence $u>\pi u>\pi^{2} u>\ldots$ would contradict that $\leq$ is a well-order.

Let $K$ be a field and denote by $K[X]=K$ Mon the ring of polynomials in the variables in $X$, or, equivalently, the monoid algebra over $K$ of Mon. The action of $\Pi$ on Mon extends to an action on $K[X]$ by means of ring endomorphisms preserving 1. For a non-zero $f \in K[X]$ denote by $\ln (f) \in$ Mon the largest monomial with a non-zero coefficient in $f$. As the action of $\Pi$ preserves the (strict) monomial order, we have $\operatorname{lm}(\pi f)=\pi \operatorname{lm}(f)$ in addition to the usual properties of $\operatorname{lm}$. In other words, the map $\operatorname{lm}$ from $K[X] \backslash\{0\}$ to Mon is $\Pi$-equivariant, and this motivates the terminology in the following definition.

Definition 2.1. Let $I$ be a $\Pi$-stable ideal in $K[X]$. Then a $\Pi$-Gröbner basis, or equivariant Gröbner basis if $\Pi$ is clear from the context, of $I$ is a subset $B$ of $I$ with the property that for any $f \in I$ there exists a $g \in B$ and a $\pi \in \Pi$ with $\operatorname{lm}(\pi g) \mid \operatorname{lm}(f)$.

The set $B$ is an equivariant Gröbner basis of $I$ if and only if the union $\Pi B=$ $\{\pi g \mid \pi \in \Pi, g \in B\}$ of the $\Pi$-orbits of elements of $B$ is an ordinary Gröbner basis of $I$ (except that it will typically not be finite). Then, in particular, $\Pi B$ generates $I$ as an ideal; and we also say that $B$ generates $I$ as a $\Pi$-stable ideal.

We do not require that an equivariant Gröbner basis be finite, but finite ones will of course be the most useful ones to us. To formulate a criterion guaranteeing the existence of finite equivariant Gröbner bases we define the $\Pi$-divisibility relation on Mon by $\left.u\right|_{\Pi} v$ if and only if there exists a $\pi \in \Pi$ such that $\pi u$ divides $v$. This relation is reflexive (take $\pi=1$ ), transitive (if $v=u^{\prime} \cdot \pi u$ and $w=v^{\prime} \cdot \sigma v$, then $w=\left(v^{\prime} \sigma u^{\prime}\right) \cdot(\sigma \pi) u$ ), and antisymmetric (if $\pi u \mid v$ and $\sigma v \mid u$ then $u \leq \pi u \leq v$ and $v \leq \sigma v \leq u$ so that $u=v)$. 
Proposition 2.2 ([HS12]). Every $\Pi$-stable ideal $I \subseteq K[X]$ has a finite $\Pi$ Gröbner basis if and only if $\left.\right|_{\Pi}$ is a well-partial-order.

PROOF. For the "only if" part observe that if $u_{1}, u_{2}, \ldots$ were a bad sequence of monomials, then the $\Pi$-stable ideal generated by them, i.e., the smallest $\Pi$-stable ideal containing them, would not have a finite equivariant Gröbner basis. For the "if" part let $I$ be a $\Pi$-stable ideal in $K[X]$. Let $M$ denote the set of $\left.\right|_{\Pi \text {-minimal }}$ elements of $\{\operatorname{lm}(f) \mid f \in I \backslash\{0\}\}$. As $\left.\right|_{\Pi}$ is a well-partial-order, $M$ is finite, say $M=\left\{u_{1}, \ldots, u_{p}\right\}$. Choose $f_{1}, \ldots, f_{p} \in I \backslash\{0\}$ with $\operatorname{lm}\left(f_{i}\right)=u_{i}$. Then $\left\{f_{1}, \ldots, f_{p}\right\}$ is a $\Pi$-Gröbner basis of $I$.

The main example that we shall use has $X:=\left\{x_{i j} \mid i \in[k], j \in \mathbb{N}\right\}$ and $\Pi:=$ $\operatorname{Inc}(\mathbb{N})$, the monoid of maps $\mathbb{N} \rightarrow \mathbb{N}$ that are strictly increasing in the standard order on $\mathbb{N}$. This monoid acts on $X$ by $\pi x_{i j}=x_{i \pi(j)}$; the action extends multiplicatively to an action on Mon and linearly to an action by ring endomorphisms on the polynomial ring $R:=K[X]=K\left[\left(x_{i j}\right)_{i j}\right]$. There exist monomial orders $\leq$ for which $u<v$ implies $\pi u<\pi v$; for instance, the lexicographic order with $x_{i j}<x_{i^{\prime} j^{\prime}}$ if $i<i^{\prime}$ or $i=i^{\prime}$ and $j<j^{\prime}$.

TheOrem 2.3 (Coh87, HS12). Fix a natural number $k$. Then any $\operatorname{Inc}(\mathbb{N})$ stable ideal $I$ in the ring $K\left[x_{i j} \mid i \in[k], j \in \mathbb{N}\right]$ has a finite $\operatorname{Inc}(\mathbb{N})$-Gröbner basis with respect to any monomial order preserved by $\operatorname{Inc}(\mathbb{N})$. In particular, any $\operatorname{Inc}(\mathbb{N})$ stable ideal I in that ring is generated, as an ideal, by finitely many $\operatorname{Inc}(\mathbb{N})$-orbits of polynomials.

Proof. By Proposition 2.2 it suffices to prove that $\left.\right|_{\operatorname{Inc}(\mathbb{N})}$ is a well-partialorder. To this end, we shall apply Higman's Lemma to $S=\mathbb{Z}_{>0}^{k}$ with the componentwise partial order, which is a well-partial-order by Dickson's Lemma. Encode a monomial $u$ in the variables $x_{i j}$ as a word $\left(s_{1}, \ldots, s_{p}\right)$ in $S^{*}$ as follows: $p$ is the largest value of the column index $j$ for which some variable $x_{i j}$ appears in $u$, and $\left(s_{j}\right)_{i}$ is the exponent of $x_{i j}$ in $u$. Now given any sequence $u_{1}, u_{2}, \ldots$ of monomials, by Higman's Lemma there exist indices $m<l$ such that the sequences $s, s^{\prime}$ encoding $u_{m}, u_{l}$ satisfy $s \leq s^{\prime}$. This means that there exists a strictly increasing map $\pi:[p] \rightarrow\left[p^{\prime}\right]$, with $p, p^{\prime}$ the lengths of $s, s^{\prime}$, such that $s_{j} \leq s_{\pi(j)}^{\prime}$ for all $j \in[p]$. Extend $\pi$ in any manner to a strictly increasing map $\mathbb{N} \rightarrow \mathbb{N}$. Then the exponent of any variable $x_{i j}$ in $\pi u_{m}$ equals 0 if $j \notin \pi([p])$ and $\left(s_{\pi^{-1} j}\right)_{i} \leq\left(s_{j}^{\prime}\right)_{i}$ otherwise. This proves that $\pi u_{m} \mid u_{l}$, as desired.

The second statement in Theorem 2.3 has several consequences. One is that any ascending chain $I_{1} \subseteq I_{2} \subseteq$. . of Inc $(\mathbb{N})$-stable ideals in $R$ stabilises at some finite in$\operatorname{dex} n: I_{n}=I_{n+1}=\ldots$; we express this fact by saying that $R$ is $\operatorname{Inc}(\mathbb{N})$-Noetherian. This implies that $R$ is $\operatorname{Sym}(\infty)$-Noetherian, where the group $\operatorname{Sym}(\infty):=\bigcup_{j \in \mathbb{N}} \operatorname{Sym}([j])$ is obtained by embedding $\operatorname{Sym}([j])$ into $\operatorname{Sym}([j+1])$ as the stabiliser of $j+1$ and where $\pi \in \operatorname{Sym}(\infty)$ acts on $x_{i j}$ by $\pi x_{i j}=x_{i \pi(j)}$. Indeed, the $\operatorname{Sym}(\infty)$-orbit of any polynomial $f$ contains the $\operatorname{Inc}(\mathbb{N})$-orbit of $f$, and hence any $\operatorname{Sym}(\infty)$-stable ideal is also $\operatorname{Inc}(\mathbb{N})$-stable. Note that one can also replace the countable group $\operatorname{Sym}(\infty)$ by the uncountable group of all permutations of $\mathbb{N}$, because the two have exactly the same orbits on $R$.

EXAMPLE 2.4. In contrast to these beautiful positive results, consider the set $X=\left\{y_{i j} \mid i, j \in \mathbb{N}\right\}$ with $\Pi=\operatorname{Inc}(\mathbb{N})$-action given by $\sigma y_{i j}=y_{\sigma(i) \sigma(j)}$. We claim 
that $\left.\right|_{\Pi}$ is not a well-partial-order. Indeed, consider the sequence of monomials

$$
y_{12} y_{21}, y_{12} y_{23} y_{31}, \ldots
$$

encoding directed cycles on two, three, etc. vertices. Any $\pi \in \operatorname{Inc}(\mathbb{N})$ maps such a monomial to a monomial representing another directed cycle of the same length. Since no larger cycle contains a smaller cycle as a subgraph, this is a bad sequence of monomials. The same argument shows that $K[X]$ is not $\operatorname{Sym}(\infty)$-Noetherian. Similar counterexamples exist for the action of $\operatorname{Sym}(\infty) \times \operatorname{Sym}(\infty)$ on $X$ given by $(\pi, \sigma) y_{i j}=y_{\pi(i) \sigma(j)}$.

We now return to the general setting of equivariant Gröbner bases, without the assumption that $\left.\right|_{\Pi}$ is a well-partial-order. These bases can sometimes be computed by a П-equivariant version of Buchberger's algorithm. The halting criterion in this equivariant Buchberger algorithm is the following equivariant version of Buchberger's criterion involving $S$-polynomials.

Proposition 2.5 (Equivariant Buchberger criterion). Let $B$ be a subset of a $\Pi$-stable ideal $I$ in $K[X]$. Then $B$ is a $\Pi$-Gröbner basis of $I$ if and only if for all $f, g \in B$ and all $\sigma, \tau \in \Pi$ the ordinary $S$-polynomial $S(\sigma f, \tau g)$ gives remainder 0 upon division by $\Pi B$.

This criterion follows immediately from the ordinary Buchberger criterion applied to $\Pi B$-indeed, while most textbooks assume a finite number of variables, division-with-remainder and Buchberger's criterion apply to infinitely many variables as well; the crucial ingredient is the fact that the monomial order is a wellorder. Unfortunately, since $\Pi$ is typically infinite, checking whether $B$ is an equivariant Gröbner basis using the equivariant Buchberger criterion may be an infinite task, even when $B$ is finite. But in many cases of interest this task can be reduced to a finite task as follows.

Assume, first, that for any two polynomials $f, g \in K[X]$ the Cartesian product $\Pi f \times \Pi g$ of the $\Pi$-orbits of $f$ and $g$ is the union of finitely many diagonal orbits $\Pi\left(\sigma_{i} f, \tau_{i} g\right)=\left\{\left(\pi \sigma_{i} f, \pi \tau_{i} g\right) \mid \pi \in \Pi\right\}, i=1, \ldots, r$, where $r \in \mathbb{N}$ and $\sigma_{1}, \tau_{1}, \ldots, \sigma_{r}, \tau_{r} \in \operatorname{Inc}(\mathbb{N})$ are allowed to depend on $f, g$. Then we would like to check only whether the $S$-polynomials $S\left(\sigma_{i} f, \tau_{i} g\right)$ reduce to zero upon division by $\Pi B$, and conclude that all $S(\sigma f, \tau g)$ reduce to zero. For this we would like that $S\left(\pi \sigma_{i} f, \pi \tau_{i} g\right)=\pi S\left(\sigma_{i} f, \tau_{i} g\right)$, because letting $\pi$ act on the reduction of $S\left(\sigma_{i} f, \tau_{i} g\right)$ to zero yields a reduction of $S\left(\pi \sigma_{i} f, \pi \tau_{i} g\right)$ to zero. This desired П-equivariance of $S$-polynomials does not follow from the assumptions so far, but it does follow if we make the further assumption that each $\pi \in \Pi$ preserves least common multiples, i.e., that $\operatorname{lcm}(\pi u, \pi v)=\pi \operatorname{lcm}(u, v)$ for all $u, v \in$ Mon. This is, in particular, the case if $\pi$ maps variables to variables.

TheOREm 2.6. Assume that Cartesian products $\Pi f \times \Pi g$ of $\Pi$-orbits on $K[X]$ are unions of finitely many diagonal $\Pi$-orbits, and assume that $\Pi$ preserves least common multiples of monomials. Let $S$ be a finite subset of $K[X]$ and consider the following algorithm:

(1) Set $B:=S$ and $P:=\left(\begin{array}{l}S \\ 2\end{array}\right) \cup\{(f, f) \mid f \in S\}$, where $\left(\begin{array}{l}S \\ 2\end{array}\right)$ is the set of pairs of distinct elements from $S$;

(2) If $P=\emptyset$, then stop, otherwise pick $(f, g) \in P$ and remove it from $P$.

(3) Choose $r \in \mathbb{N}, \sigma_{1}, \tau_{1}, \ldots, \sigma_{r}, \tau_{r} \in \Pi$ such that $\Pi f \times \Pi g=\bigcup_{i=1}^{r} \Pi\left(\sigma_{i} f, \tau_{i} g\right)$. 
(4) For each $i=1, \ldots, r$ do the following: reduce $S\left(\sigma_{i} f, \tau_{i} g\right)$ modulo $\Pi B$, and if the remainder $h$ is non-zero, then add $h$ to $B$ and consequently add $B \times\{h\}$ to $P$.

(5) Return to step 2.

If and when this algorithm terminates, then $B$ is a $\Pi$-Gröbner basis for the $\Pi$-stable ideal generated by $S$. Moreover, if $\left.\right|_{\Pi}$ is a well-partial-order, then this algorithm does terminate.

As argued above, all but the last sentence of this theorem follows from the ordinary Buchberger criterion. The last sentence follows as for the ordinary Buchberger algorithm: if the algorithm does not terminate, then an infinite number of non-zero remainders $h_{1}, h_{2}, \ldots$ are added. If $\left.\right|_{\Pi}$ is a well-partial-order, then there exist $i<j$ with $\left.\operatorname{lm}\left(h_{i}\right)\right|_{\Pi} \operatorname{lm}\left(h_{j}\right)$, which means that $h_{j}$ was not reduced with respect to $h_{i}$, a contradiction.

One point to stress is that in initialising the pair set $P$ also pairs of two identical polynomials $(f, f), f \in S$ need to be added; and that similarly, when adding a remainder $h$ to $B$, also the pair $(h, h)$ needs to be added to $P$. Indeed, already the $\Pi$-stable ideal generated by a single polynomial can be interesting, as the following example shows.

Example 2.7. Let $X=\left\{x_{i} \mid i \in \mathbb{N}\right\} \cup\left\{y_{i j} \mid i, j \in \mathbb{N}, i>j\right\}$ and let $\Pi=\operatorname{Inc}(\mathbb{N})$ act on $X$ by $\pi x_{i}=x_{\pi(i)}$ and $\pi y_{i j}=y_{\pi(i) \pi(j)}$. Set $S:=\left\{y_{21}-x_{2} x_{1}\right\}$ and let $I$ denote the $\Pi$-stable ideal generated by $S$. We would like to compute an equivariant Gröbner basis of the elimination ideal $I \cap K\left[y_{i j} \mid i>j\right]$. To this end, we choose the lexicographic monomial order with $x_{1}<x_{2}<\ldots$ and $y_{i j}<y_{k l}$ if either $i<k$ or $i=k$ and $j<l$, and with $y_{k l}<x_{i}$ for all $i, k, l$. Note that $\Pi$ preserves the strict monomial order and least common multiples.

To apply the equivariant Buchberger algorithm we must further check that Cartesian products of $\Pi$-orbits on $K[X]$ are finite unions of diagonal $\Pi$-orbits. For this, let $f, g$ be elements of $K[X]$ and let $p, q$ be such that all variables in $f, g$ have indices contained in $[p],[q]$, respectively. Then $\sigma f, \tau g$ depend only on the restrictions of $\sigma$ and $\tau$ to $[p],[q]$ respectively. Enumerate all (finitely many) pairs $\left(\sigma_{i}:[p] \rightarrow \mathbb{N}, \tau_{i}:[q] \rightarrow \mathbb{N}\right), i=1, \ldots, r$ of increasing maps for which the union of $\operatorname{im}\left(\sigma_{i}\right)$ and $\operatorname{im}\left(\tau_{i}\right)$ equals some interval $[t]=\{1, \ldots, t\}$, necessarily with $t \leq p+q$. Extend these $\sigma_{i}$ and $\tau_{i}$ arbitrarily to elements of $\Pi$. We claim that for any pair $\sigma, \tau \in \Pi$ we have $(\sigma f, \tau g)=\left(\pi \sigma_{i} f, \pi \tau_{i} g\right)$ for some $i \in[r]$ and some $\pi \in \Pi$. Indeed, there exists a unique $i$ for which there exists an (again, unique) increasing map $\pi:[t]=\operatorname{im}\left(\sigma_{i}\right) \cup \operatorname{im}\left(\tau_{i}\right) \rightarrow \sigma([p]) \cup \tau([q])$ such that the restrictions of $\sigma, \tau$ to $[p],[q]$ equal the restrictions of $\pi \circ \sigma_{i}, \pi \circ \tau_{i}$ to $[p],[q]$, respectively. Extend $\pi$ in any manner to an element of $\Pi$ and we find $(\sigma f, \tau g)=\left(\pi \sigma_{i} f, \pi \tau_{i} g\right)$, as desired.

This means that we can apply the equivariant Buchberger algorithm, but without the guarantee that it terminates, since $\left.\right|_{\Pi}$ is not a well-partial-order (adapt Example 2.4 to see this). It turns out the algorithm does terminate, though, and yields the following equivariant Gröbner basis (after self-reduction):

$$
\begin{aligned}
B= & \left\{x_{1} x_{2}-y_{21},\right. \\
& x_{3} y_{21}-x_{2} y_{31}, x_{3} y_{21}-x_{1} y_{32}, x_{2} y_{31}-x_{1} y_{32}, \\
& x_{1}^{2} y_{32}-y_{31} y_{21}, \\
& \left.y_{43} y_{21}-y_{41} y_{32}, y_{42} y_{31}-y_{41} y_{32} \cdot\right\}
\end{aligned}
$$


Since the monomial order is an elimination order, we conclude that $I \cap K\left[y_{i j} \mid i>j\right]$ has a $\Pi$-Gröbner basis given by the last two binomials. In particular, that ideal is generated, as an $\operatorname{Inc}(\mathbb{N})$-stable ideal, by these binomials. The result that we have just proved by computer has first appeared as a theorem in dLST95. This example gives the ideal of the so-called second hypersimplex, or, with a slight modification, of the Gaussian one-factor model. The $k$-factor model for $k=2$ and higher will be the subject of Chapter 6 . 



\section{CHAPTER 3}

\section{Equivariant Noetherianity}

In this chapter we establish a number of constructions of equivariantly Noetherian rings and spaces. For some of the material see Dra10.

Given a ring $R$ (always commutative, with 1 ) and a monoid $\Pi$ with a left action on $R$ by means of (always unital) endomorphisms we say that $R$ is $\Pi$-Noetherian, or equivariantly Noetherian if $\Pi$ is clear from the context, if every chain $I_{1} \subseteq I_{2} \subseteq \ldots$ of $\Pi$-stable ideals in $R$ eventually stabilises, that is, if there exists an $n \in \mathbb{N}$ for which $I_{n}=I_{n+1}=\ldots$. This is equivalent to the condition that any $\Pi$-stable ideal $I$ is generated, as an ideal, by finitely many $\Pi$-orbits $\Pi f_{1}, \ldots, \Pi f_{s}$. We then say that $f_{1}, \ldots, f_{s}$ generate $I$ as a $\Pi$-stable ideal.

We have seen a major example in Chapter 2, namely, for any fixed natural number $k$ and any field $K$ the $\operatorname{ring} K\left[x_{i j} \mid i \in[k], j \in \mathbb{N}\right]$ with its action of $\operatorname{Inc}(\mathbb{N})$ on the second index is $\operatorname{Inc}(\mathbb{N})$-Noetherian.

There are several constructions of new equivariantly Noetherian rings from existing ones. The first and most obvious is that if $R$ is $\Pi$-Noetherian and $I \subseteq R$ is a $\Pi$-stable ideal, then $R / I$ is $\Pi$-Noetherian: any chain of $\Pi$-stable ideals in $R / I$ lifts to a chain of $\Pi$-stable ideals in $R$ containing $I$, and the first chain stabilises exactly when the second chain does.

A second construction takes a ח-Noetherian ring $R$ to the polynomial ring $R[x]$ in a variable $x$, where $\Pi$ acts only on the coefficients from $R$. The standard proof of Hilbert's Basis Theorem, say from Lan65, generalises word by word from trivial $\Pi$ to general $\Pi$.

It is not true, in general, that a subring of an equivariantly Noetherian ring is equivariantly Noetherian. Indeed, this is already not true for ordinary Noetherianity, where $\Pi$ is the trivial monoid. However, the following construction proves that certain well-behaved subrings of equivariantly Noetherian rings are again equivariantly Noetherian. Suppose that $S$ is a subring of $R$ with the property that $R$ splits as a direct sum $S \oplus M$ of $S$-modules. If $J$ is an ideal in $S$ and $I$ is the ideal in $R$ generated by $J$, then we claim that $S \cap I=J$-indeed, any element $f$ of $S \cap I$ can be written as $f=\sum_{i} f_{i} g_{i}$ with the $f_{i}$ elements of $J$ and the $g_{i}$ elements of $R$. Applying the $S$-linear projection $\pi: R \rightarrow S$ along $M$ to both sides yields $f=\sum_{i} f_{i} \pi\left(g_{i}\right) \in J$, as claimed. If, moreover, the monoid $\Pi$ acts on $R$ and stabilises $S$, then $I$ is $\Pi$-stable if $J$ is. We conclude that if $R$ is $\Pi$-Noetherian, then any chain $I_{1} \subseteq I_{2} \subseteq \ldots$ of $\Pi$-stable ideals in $S$ generates such a chain $J_{1} \subseteq J_{2} \subseteq \ldots$ in $R$, and $J_{n}=J_{n+1}=\ldots$ implies that $I_{n}=J_{n} \cap S=J_{n+1} \cap S=I_{n+1}=\ldots$; so $S$ is $\Pi$-Noetherian.

A particularly important example of this situation is the following proposition, due to Kuttler. Suppose that a group $H$ acts on $R$ by means of ring automorphisms, and that the action of $H$ commutes with that of $\Pi$, i.e., for every $\pi \in \Pi$ and $h \in H$ 
and $f \in R$ we have $\pi h f=h \pi f$. Then the ring $R^{H}:=\{f \in R \mid H f=\{f\}\}$ of $H$-invariant elements of $R$ is stable under the action of $\Pi$.

Proposition 3.1. If on the one hand $R$ is $\Pi$-Noetherian and on the other hand $R$ splits as a direct sum of irreducible $\mathbb{Z} H$-modules, then $R^{H}$ is also П-Noetherian.

Proof. By the discussion preceding the proposition, we need only prove that $R$ splits as a direct sum $R^{H} \oplus M$ of $R^{H}$-modules. For this, split $R$ as a direct sum $\oplus_{i} M_{i}$ of irreducible $\mathbb{Z} H$-modules $M_{i}$. Then $R^{H}$ is the direct sum of the $M_{i}$ with trivial $H$-action, and we set $M$ equal to the direct sum of the $M_{i}$ with non-trivial $H$-action. We want to show that $f M_{i} \subseteq M$ for every $f \in R^{H}$ and every $M_{i} \subseteq M$. To this end, let $\rho: R \rightarrow R^{H}$ be the projection along $M$ and consider the map $M_{i} \rightarrow R^{H}$ sending $m$ to $\rho(f m)$. By invariance of $f$ this map is $H$-equivariant, and by irreducibility of $M_{i}$ its kernel is either $\{0\}$ or all of $M_{i}$. But in the first case, the non-trivial $H$-module $M_{i}$ would be embedded into the trivial $H$-module $R^{H}$, which is impossible. Hence that kernel is all of $M_{i}$, and $f M_{i} \subseteq M$.

In our applications, $R$ will typically be an algebra over some field $K$, and $H$ will act $K$-linearly. Then it suffices that $R$ splits as a direct sum of irreducible $\mathrm{KH}$-modules (as one can infer from the proposition by replacing $H$ by the group $\left.K^{*} \times H\right)$.

We give several applications of this proposition. First, we have seen in Example 2.4 that the ring of polynomials in the entries $y_{i j}$ of an $\mathbb{N} \times \mathbb{N}$-matrix is not Inc(N)-Noetherian. The following corollaries show that interesting quotients of such rings are $\operatorname{Inc}(\mathbb{N})$-Noetherian.

Corollary 3.2. Let $k$ be a natural number. Consider the homomorphism $\psi: K\left[y_{\mathbf{m}} \mid \mathbf{m} \in \mathbb{N}^{k}\right] \rightarrow K\left[x_{i j} \mid i \in[k], j \in \mathbb{N}\right]$ sending $y_{\mathbf{m}}$ to $\prod_{i=1}^{k} x_{i, m_{i}}$. The kernel of $\psi$ is generated by finitely many $\operatorname{Inc}(\mathbb{N})$-orbits of polynomials, and the quotient $K\left[\left(y_{\mathbf{m}}\right)_{\mathbf{m}}\right] / \operatorname{ker} \psi$ is $\operatorname{Inc}(\mathbb{N})$-Noetherian.

In more geometric language, that quotient is the coordinate ring of $k$-dimensional infinite-by-infinite-by-... -by-infinite tensors of rank one.

Proof. The first statement follows from the standard fact that the ideal of the variety of rank-one tensors is generated by the quadrics $y_{m_{0} m_{1}} y_{m_{0}^{\prime} m_{1}^{\prime}}-y_{m_{0} m_{1}^{\prime}} y_{m_{0}^{\prime} m_{1}}$, where $m_{0}, m_{0}^{\prime}$ are multi-indices of length equal to some $\ell \leq k$ and $m_{1}, m_{1}^{\prime}$ are multi-indices of length $k-\ell$. The entries of the multi-indices $m_{0}, m_{0}^{\prime}, m_{1}, m_{1}^{\prime}$ taken together form a set of cardinality at most $2 k$, and this implies that each quadric of the form above is obtained by applying some element of $\operatorname{Inc}(\mathbb{N})$ to one of the finitely many such quadrics with all indices in the interval $[2 k]$.

The second statement follows from the proposition (or rather the discussion preceding it): the quotient is isomorphic, as a $\operatorname{ring}$ with $\operatorname{Inc}(\mathbb{N})$-action, to the subring $S=\operatorname{im} \psi$ of $K\left[x_{i j} \mid i \in[k], j \in \mathbb{N}\right]$. The ring $S$ consists of all monomials in the $x_{i j}$ that involve equally many variables, counted with their exponents, from all of the $k$ rows of the $k \times \mathbb{N}$-matrix $\left(x_{i j}\right)_{i j}$. If one writes $M$ for the vector space complement of $S$ spanned by all other monomials, then $M$ is an $S$-module, and the fact that $K\left[\left(x_{i j}\right)_{i j}\right]$ is $\operatorname{Inc}(\mathbb{N})$-Noetherian implies that $S$ is.

Alternatively, if $K$ is infinite, then one can characterise $S$ as the set of $H$ invariants, where $H$ is the subgroup of $\left(K^{*}\right)^{k}$ consisting of $k$-tuples with product 1 and where $h$ acts on $x_{i j}$ by $h x_{i j}=h_{i} x_{i j}$. Each monomial outside $S$ spans an 
irreducible, non-trivial $H$-module, and the proposition implies that $S$ is $\operatorname{Inc}(\mathbb{N})$ Noetherian.

A substantial generalisation of Corollary 3.2, which applies to a wide class of monomial maps into $K\left[\left(x_{i j}\right)_{i j} \mid i \in[k], j \in \mathbb{N}\right.$ ], is proved in DEKL13. For stabilisation of appropriate lattice ideals, see HMdC13.

The next corollary shows that determinantal quotients of the coordinate ring of infinite-by-infinite matrices are $\operatorname{Inc}(\mathbb{N})$-Noetherian, provided that the field has characteristic zero.

Corollary 3.3. For any natural number $k$ and any field $K$ of characteristic zero, the quotient of the ring $K\left[y_{i j} \mid i, j \in \mathbb{N}\right]$ by the ideal $I_{k}$ generated by all $(k+1) \times(k+1)$-minors of the matrix $\left(y_{i j}\right)_{i j}$ is $\operatorname{Inc}(\mathbb{N})$-Noetherian.

Note that the set of these determinants is the union of finitely many $\operatorname{Inc}(\mathbb{N})$ orbits of equations, so that the corollary implies that any $\operatorname{Inc}(\mathbb{N})$-stable ideal containing $I_{k}$ is generated by finitely many $\operatorname{Inc}(\mathbb{N})$-orbits.

Proof. Let the group $H=\mathrm{GL}_{k}$ act on the ring $K\left[x_{i l} \mid i \in \mathbb{N}, l \in[k]\right]$ by $h x_{i l}:=(x h)_{i l}$, where $x h$ is the product of the $\mathbb{N} \times k$-matrix $x$ with variable entries and the $k \times k$-matrix $h$. Similarly, let $H$ act on the $\operatorname{ring} K\left[z_{l j} \mid l \in[k], j \in \mathbb{N}\right]$ by $h z_{l j}:=\left(h^{-1} z\right)_{l j}$. Note that both actions commute with the action of $\operatorname{Inc}(\mathbb{N})$ on the indices $i, j$, respectively. Let $R$ be the polynomial ring $K\left[x_{i l}, z_{l j} \mid i, j \in \mathbb{N}, l \in[k]\right]$, equipped with the natural $\operatorname{Inc}(\mathbb{N})$-action and $H$-action. Classical invariant theory tells us that rings, like $R$, on which $H$ acts as an algebraic group split into a direct sum of irreducible $K H$-modules; here we use that char $K=0$. So we may apply Proposition 3.1.

The First Fundamental Theorem [GW09, Theorem 5.2.1] for $H$ states that the algebra $R^{H}$ of $H$-invariant elements of the ring $R$ is generated by all pairings $p_{i j}:=\sum_{l} x_{i l} z_{l j}=(x z)_{i j}$. The Second Fundamental Theorem [GW09, Theorem 12.2.12] states that the kernel of the homomorphism $K\left[\left(y_{i j}\right)_{i j}\right] \mapsto R$ determined by $y_{i j} \mapsto p_{i j}$ is precisely $I_{k}$. Thus the quotient by $I_{k}$ is isomorphic, as a $K$-algebra with $\operatorname{Inc}(\mathbb{N})$-action, to $R^{H}$. This proves the corollary.

Similar results are obtained by taking other rings with group actions where the invariants and the polynomial relations among them are known. Here is an example, which first appeared in Dra10.

COROLlary 3.4. For any natural number $k$ and any field $K$ of characteristic zero, the kernel of the homomorphism $\psi: K\left[y_{\mathbf{m}} \mid \mathbf{m} \in \mathbb{N}^{k}, m_{1}<\ldots<m_{k}\right] \rightarrow$ $K\left[x_{i j} \mid i \in[k], j \in \mathbb{N}\right]$ sending $y_{\mathbf{m}}$ to the determinant of $x[\mathbf{m}]$, the $k \times k$-submatrix of $x$ obtained by taking the columns indexed by $\mathbf{m}$, is generated by finitely many $\operatorname{Inc}(\mathbb{N})$-orbits; and the quotient of $K\left[\left(y_{\mathbf{m}}\right)_{\mathbf{m}}\right]$ by $\operatorname{ker} \psi$ is $\operatorname{Inc}(\mathbb{N})$-Noetherian.

Proof. Let $H=\mathrm{SL}_{k}$, the group of $k \times k$-matrices of determinant 1, act on $K\left[\left(x_{i j}\right)_{i j}\right]$ by $h x_{i j}=\left(h^{-1} x\right)_{i j}$. The First Fundamental Theorem for $\mathrm{SL}_{n}$ says that the $k \times k$-minors of $x$ generate the invariant ring of $H$, and the Second Fundamental Theorem says that the Plücker relations among those determinants, which can be covered by finitely many $\operatorname{Inc}(\mathbb{N})$-orbits, generate the ideal of all relations. Now proceed as in the previous case. 
We remark that Alexei Krasilnikov showed that the Noetherianity of this corollary does not hold when char $K=2$ and $k=2$. However, a weaker form of Noetherianity, which we introduce now, does hold.

Let $X$ be a topological space equipped with a right action of a monoid $\Pi$ by means of continuous maps $X \rightarrow X$. Then we call $X$ equivariantly Noetherian, or $\Pi$-Noetherian, if every chain $X=X_{0} \supseteq X_{1} \supseteq X_{2} \supseteq \ldots$ of closed, $\Pi$-stable subsets stabilises. If $R$ is a $K$-algebra with a left action of $\Pi$ by means of $K$-algebra endomorphisms, then for any $K$-algebra $A$ the set $X:=R(A):=\operatorname{Hom}_{K}(R, A)$ of $A$-valued points of $R$ is a topological space with respect to the Zariski topology in which closed sets are defined by the vanishing of elements of $R$. Moreover, the monoid $\Pi$ acts from the right on $R(A)$ by $(p \pi)(r)=p(\pi r)$. If $R$ is $\Pi-N o e t h e r i a n$, then $R(A)$ is $\Pi$-Noetherian in the topological sense. Conversely, if $R(A)$ is $\Pi$ Noetherian in the topological sense for every $K$-algebra $A$, then $R$ is $\Pi$-Noetherianindeed, just take $A$ equal to $R$, so that the map that takes closed sets to vanishing ideals is a bijection. However, topological Noetherianity of, say, $R(K)$ does not necessarily imply Noetherianity of $R$. An example of this phenomenon is given by Krasilnikov's example: the $\operatorname{ring} K[\operatorname{det} x[i, j] \mid i, j \in \mathbb{N}, i<j]$, where $x$ is a $[2] \times \mathbb{N}$-matrix of variables, is not $\operatorname{Inc}(\mathbb{N})$-Noetherian if char $K=2$, but its set of $K$-valued points is -indeed, this set of points is the image of $K^{[2] \times \mathbb{N}}$ under the $\operatorname{Inc}(\mathbb{N})$-equivariant map sending a matrix to the vector of its $2 \times 2$-determinants. Since $K^{[2] \times \mathbb{N}}$ is $\operatorname{Inc}(\mathbb{N})$-Noetherian, so is its image.

More generally, П-equivariant images of $\Pi$-Noetherian topological spaces are $\Pi$-Noetherian, and so are П-stable subsets with the induced topology. Another construction that we shall make much use of is the following.

Proposition 3.5. Let $G$ be a group with a right action by homeomorphisms on a topological space $X$. Let $\Pi$ be a submonoid of $G$ and let $Z$ be a $\Pi$-stable subset of $X$. Assume that $Z$ is $\Pi$-Noetherian with the induced topology. Then $Y:=Z G=\bigcup_{g \in G} Z g \subseteq X$ is $G$-Noetherian with the induced topology.

Proof. Let $Y=Y_{1} \supseteq Y_{2} \supseteq Y_{3} \supseteq \ldots$ be a chain of $G$-stable closed subsets of $Y$. Then each $Z_{i}:=Y_{i} \cap Z$ is $\Pi$-stable and closed, hence by $\Pi$-Noetherianity of $Z$ there exists an $n$ with $Z_{n}=Z_{n+1}=\ldots$. By definition of $Y$, for each $y \in Y_{i}$ there exist a $g \in G$ and a $z \in Z$ with $y=z g$, and by $G$-stability of $Y_{i}$ we have $z=y g^{-1} \in Z_{i}$. This means that $Y_{i}$ can be recovered from $Z_{i}$ as $Y_{i}=Z_{i} G$, and hence the chain $Y_{1} \supseteq Y_{2} \supseteq Y_{3} \supseteq \ldots$ stabilises at $Y_{n}$, as well. 


\section{CHAPTER 4}

\section{Chains of varieties}

In the remainder of these notes we study various chains of interesting embedded finite-dimensional varieties, for which we want to prove that from some member of the chain on, all equations for later members come from those of earlier members by applying symmetry. To use the infinite-dimensional techniques from the previous chapters, we first pass to a projective limit, prove that the limit is defined by finitely many orbits of equations, and from this fact we derive the desired result concerning the finite-dimensional varieties. In this short chapter we set up the required framework for this, again without trying to be as general as possible. Most of this material is from Dra10.

Thus let $K$ be a field and let $R_{1}, R_{2}, \ldots$ be commutative $K$-algebras with 1 . The algebra $R_{i}$ plays the role of coordinate ring of the ambient space of the $i$-th variety in our chain. Assume that the $R_{i}$ are linked by (unital) ring homomorphisms $\iota_{i}: R_{i} \rightarrow R_{i+1}$ and $\pi_{i}: R_{i+1} \rightarrow R_{i}$ satisfying $\pi_{i} \circ \iota_{i}=1_{R_{i}}$. Then we can form the $K$-algebra $R_{\infty}:=\bigcup_{i \in \mathbb{N}} R_{i}$ with respect to the inclusions $\iota_{i}$; the use of the $\pi_{i}$ will become clear later.

Suppose, next, that are given ideals $I_{i} \subseteq R_{i}$ such that $\pi_{i}$ maps $I_{i+1}$ into $I_{i}$ and $\iota_{i}$ maps $I_{i}$ into $I_{i+1}$. The ideal $I_{i}$ plays the role of defining ideal of the $i$-th variety in our chain. Writing $S_{i}:=R_{i} / I_{i}$ we find that the $\iota_{i}, \pi_{i}$ induce inclusions $S_{i} \rightarrow S_{i+1}$ and surjections $S_{i+1} \rightarrow S_{i}$, respectively, and we set $I_{\infty}:=\bigcup_{i} I_{i}$ and $S_{\infty}:=\bigcup_{i} S_{i}$, which also equals $R_{\infty} / I_{\infty}$.

Assume, next, that a group $G_{i}$ acts on $R_{i}$ from the left by means of $K$-algebra automorphisms, and that we are given embeddings $G_{i} \rightarrow G_{i+1}$ that render both $\iota_{i}$ and $\pi_{i}$ equivariant with respect to $G_{i}$. Suppose furthermore that each $I_{i}$ is $G_{i^{-}}$ stable, which expresses that the $i$-th variety has the same symmetries as imposed on the ambient space. We form the group $G_{\infty}:=\bigcup_{i} G_{i}$, which acts on $R_{\infty}, I_{\infty}, S_{\infty}$ by means of automorphisms.

For any $K$-algebra $A$, we write $R_{i}(A), S_{i}(A), R_{\infty}(A), S_{\infty}(A)$ for the sets of $A$ valued points of these algebras, i.e., for the set of homomorphisms $R_{i} \rightarrow A$, etc. As customary in algebraic geometry, for a $p$ in these point sets, we write $f(p)$ rather than $p(f)$ for the evaluation of $p$ on an element $f$ in the corresponding algebra. These sets are topological spaces with respect to the Zariski topology, in which closed sets are of the form $\left\{p \in R_{i}(A) \mid J(p)=\{0\}\right\}$ for some ideal $J$ in $R_{i}$, and similarly for the other algebras. On these topological spaces $G_{i}$ or $G_{\infty}$ acts by means of homeomorphisms. Our set-up so far is summarised in the diagram of Figure 4.1, where $\iota^{*}, \pi^{*}$ are the pull-backs of $\iota$ and $\pi$, respectively.

The relation $\iota^{*} \circ \pi^{*}=1$ implies that $\iota^{*}$ is surjective (and not just dominant) and that $\pi^{*}$ is injective; indeed, the latter is a closed embedding. Still, $\pi^{*}$ is needed only a bit later. 


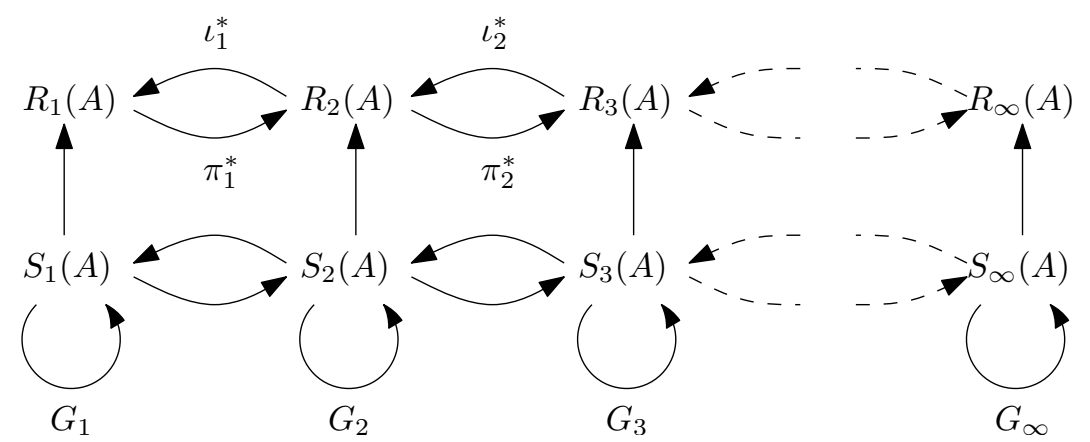

FIgURE 4.1. Chains of varieties

The topological space $R_{\infty}(A)$ is canonically the same as the projective limit, in the category of topological spaces, of the spaces $R_{i}(A)$ with their Zariski topologies: First, at the level of sets, an $A$-valued point $p$ of $R_{\infty}$ gives rise, by composition with the embeddings $R_{i} \rightarrow R_{\infty}$, to homomorphisms $p_{i}:=R_{i} \rightarrow A$ for all $i \in \mathbb{N}$. The resulting sequence $\left(p_{1}, p_{2}, \ldots\right)$ has the property that the pull-back of $\iota_{i}^{*}$ maps $p_{i+1}$

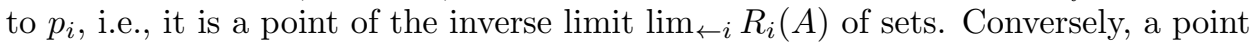
of this inverse limit gives homomorphisms $p_{i}: R_{i} \rightarrow A$ such that $p_{i+1} \circ \iota_{i}=p_{i}$, and together these define a homomorphism $R_{\infty} \rightarrow A$. Second, the projective limit topology on $R_{\infty}(A)$ is the weakest topology that renders all maps $R_{\infty}(A) \rightarrow R_{i}(A)$ continuous. This means, in particular, that sets given by the vanishing of a single element of $R_{i} \subseteq R_{\infty}$ must be closed, and so must intersections of these, which are sets given by the vanishing of an ideal in $R_{\infty}$. This shows that the projective limit topology on $R_{\infty}(A)$ has at least as many closed sets as the Zariski topology, and the converse is also clear since the maps $R_{\infty}(A) \rightarrow R_{i}(A)$ are continuous in the Zariski topology.

The basic result that we shall use throughout the rest of the notes is the following, where the use of the $\pi_{i}$ becomes apparent.

Proposition 4.1. Let $i_{0} \in \mathbb{N}$ and assume that the set $S_{\infty}(A)$ is characterised inside $R_{\infty}(A)$ by the vanishing of all $g f \in R_{\infty}$ with $g \in G_{\infty}$ and $f \in I_{i_{0}}$. Then for $i \geq i_{0}$ the set $S_{i}(A)$ is characterised by the vanishing of all functions of the form $\pi_{i} \cdots \pi_{l-1} g f$ with $l \geq i, g \in G_{l}$, and $f \in I_{i_{0}}$.

Proof. That these functions vanish on $S_{i}(A)$ follows from the inclusion $I_{i_{0}} \subseteq$ $I_{l}$, the fact that $G_{l}$ stabilises $I_{l}$, and the fact that $\pi_{j}$ maps $I_{j+1}$ into $I_{j}$. Conversely, suppose that $p_{i} \in R_{i}(A)$ is a zero of all functions in the proposition, and let $p=$ $\left(p_{1}, p_{2}, \ldots\right)$ be the point of $R_{\infty}(A)$ obtained by setting $p_{j+1}:=\pi_{j}^{*} p_{j}$ for $j \geq i$ and $p_{j}:=\iota_{j}^{*} p_{j+1}$ for $j<i$. Then $p$ is a point in $S_{\infty}(A)$ by the assumed characterisation of the latter set: any $g \in G_{\infty}$ lies in $G_{l}$ for some $l$ which we may take larger than $i$, and for $f \in I_{i_{0}}$ we have

$$
(g f)(p)=(g f)\left(p_{l}\right)=(g f)\left(\pi_{l-1}^{*} \cdots \pi_{i}^{*} p_{i}\right)=\left(\pi_{l-1} \cdots \pi_{i} g f\right)\left(p_{i}\right)=0,
$$

as desired. In particular, this means that $p_{i}$ lies in $S_{i}(A)$.

It would be more elegant to characterise $S_{i}(A)$ for $i \geq i_{0}$ as the common vanishing set of $G_{i} I_{i_{0}}$, i.e., of all functions of the form $g f$ with $f \in I_{i_{0}}$ and $g \in G_{i}$. For this we introduce an additional condition. For $l \geq i$ write $\iota_{i l}: R_{i} \rightarrow R_{l}$ for 
the composition $\iota_{l-1} \cdots \iota_{i}$ and $\pi_{l i}: R_{l} \rightarrow R_{i}$ for the composition $\pi_{i} \cdots \pi_{l-1}$. The condition that we want is:

For all indices $l, i_{0}, i_{1}$ with $l \geq i_{0}, i_{1}$ and for all $g \in G_{l}$ there exist an index $j \leq i_{0}, i_{1}$ and group elements $g_{0} \in G_{i_{0}}, g_{1} \in G_{i_{1}}$ such that

$$
\pi_{l i_{1}} g \iota_{i_{0} l}=g_{1} \iota_{j i_{1}} \pi_{i_{0} j} g_{0}
$$

holds as an equality of homomorphisms $R_{i_{0}} \rightarrow R_{i_{1}}$.

This guarantees that the functions $\pi_{l i} g f=\pi_{l i} g \iota_{i_{0} l} f$ from the proposition can be written as $g_{1} \iota_{j i} \pi_{i_{0} j} g_{0} f$ for some $j \leq i, i_{0}$ and $g_{0} \in G_{i_{0}}$ and $g_{1} \in G_{i}$. Since $I_{i_{0}}$ is $G_{i_{0}}$-stable and $\pi_{i_{0} j}$ maps $I_{i_{0}}$ into $I_{j} \subseteq I_{i_{0}}$ the latter expression is an element of $G_{i} I_{i_{0}}$.

The discussion so far concerned an arbitrary, fixed $K$-algebra $A$. In several applications we shall just take $A$ equal to $K$, and the conclusion is that the point sets $S_{i}(A)$ for $i \geq i_{0}$ are defined set-theoretically by equations coming from $I_{i_{0}}$ using symmetry. However, if one assumes that $G_{\infty} I_{i_{0}}$ generates $I_{\infty}$, then the assumption in the proposition holds for all $K$-algebras $A$, hence so does the conclusion. From this one can conclude that for $i \geq i_{0}$ the functions featuring in the proposition generate the ideal $I_{i}$. Under the additional assumption $*$ one finds that $G_{i} I_{i_{0}}$ generates the ideal $I_{i}$. We conclude this chapter with a well-known example which paves the way for the treatment of the $k$-factor model in the next chapter.

EXAmple 4.2. Fix a natural number $k$. For $n \in \mathbb{N}$ let $R_{n}$ be the polynomial ring over $K$ in the $\left(\begin{array}{c}n+1 \\ 2\end{array}\right)$ variables $y_{i j}=y_{j i}$ with $i, j \leq n$. Let $\iota_{n}$ be the natural inclusion $R_{n} \rightarrow R_{n+1}$ and let $\pi_{n}$ be the projection $R_{n+1} \rightarrow R_{n}$ mapping all variables to zero that have one or both indices equal to $n+1$. Then we have $\pi_{n} \iota_{n}=1$ as required. Let $I_{n} \subseteq R_{n}$ be the ideal of polynomials vanishing on all symmetric $n \times n$-matrices over $K$ of rank at most $k$. Then $\iota_{n}$ maps $I_{n}$ into $I_{n+1}$ since the upper-left $n \times n$ block of an $(n+1) \times(n+1)$-matrix of rank at most $k$ has itself rank at most $k$, and $\pi_{n}$ maps $I_{n+1}$ into $I_{n}$ since appending a zero last row and column to any matrix yields a matrix of the same rank. Let $G_{n}:=\operatorname{Sym}(n)$ act on $R_{n}$ by $g y_{i j}=y_{g(i), g(j)}$, and embed $G_{n}$ into $G_{n+1}$ as the stabiliser of $n+1$. Then $G_{n}$ stabilises $I_{n}$ and the maps $\pi_{n}$ and $\iota_{n}$ are $G_{n}$-equivariant. So we are in the situation discussed in this chapter.

Even the additional assumption $\$$ holds. Indeed, consider the effect of appending $l-i_{1}$ zero rows and columns to a symmetric $i_{1} \times i_{1}$-matrix, then simultaneously permuting rows and columns, and finally forgetting the last $l-i_{0}$ rows and columns of which, say, $m$ come from the zero rows and columns introduced in the first step. Set $j:=i_{1}-\left(l-i_{0}-m\right)$, which is the number of rows and columns of the original matrix surviving this operation. Then the same effect is obtained by first permuting (with $g_{1}$ ) rows and columns such that the $i_{1}-j$ rows and columns to be forgotten are in the last $i_{1}-j$ positions, then forgetting those rows and columns, then appending $l-i_{1}-m=i_{0}-j$ zero rows and columns, and finally suitably permuting rows and columns with a $g_{0} \in \operatorname{Sym}\left(i_{0}\right)$.

It is known, of course, that if $K$ is infinite, then $I_{n}$ is generated by all $(k+1) \times$ $(k+1)$-minors of the matrix $\left(y_{i j}\right)_{i j}$. This implies that $I_{\infty}$ is generated by $G_{\infty} I_{2 k+2}$; here $2 k+2$ is the smallest size where representatives of all $\operatorname{Sym}(\mathbb{N})$-orbits of minors of an infinite symmetric matrix can be seen. But conversely, if through some other method (computational or otherwise) one can prove that $I_{\infty}$ is indeed generated by $G_{\infty} I_{2 k+2}$, then by the discussion above this implies that $I_{n}$ is generated by $G_{n} I_{2 k+2}$ 
for all $n \geq 2 k+2$. Using the equivariant Gröbner basis techniques from Chapter 2 one can prove such a statement automatically for small $k$, say $k=1$ and $k=2$, much like we have done in Example 2.7. 


\section{CHAPTER 5}

\section{The independent set theorem}

To appreciate the results of this chapter - though not to understand the proofsone needs some familiarity with Markov bases and their relation to toric ideals. We formulate and prove the independent set theorem from [HS12], first conjectured in [SHS07, directly at the level of ideals.

Fix a natural number $m$, let $\Gamma$ be a subset of $2^{[m]}$ (thought of as a hypergraph with vertex set $[m]$; see Figure 5.1 for an example), and fix an infinite field $K$; what follows will, in fact, not depend on $K$. To any $m$-tuple $r=\left(r_{1}, \ldots, r_{m}\right) \in \mathbb{N}^{[m]}$ of natural numbers we associate the polynomial ring

$$
R_{r}:=K\left[y_{i_{1}, \ldots, i_{m}} \mid\left(i_{1}, \ldots, i_{m}\right) \in\left[r_{1}\right] \times \cdots \times\left[r_{t}\right]\right]
$$

in $\prod_{t \in[m]} r_{t}$-many variables, and the polynomial ring

$$
Q_{r}:=K\left[x_{F,\left(i_{t}\right)_{t \in F}} \mid F \in \Gamma \text { and } \forall t \in F: i_{t} \in\left[r_{t}\right]\right] .
$$

Furthermore, we define $I_{r} \subseteq R_{r}$ as the kernel of the homomorphism $R_{r} \rightarrow Q_{r}$ mapping $y_{\left(i_{t}\right)_{t \in[m]}}$ to $\prod_{F \in \Gamma} x_{F,\left(i_{t}\right)_{t \in F}}$. On $R_{r}, Q_{r}$ acts the group $G_{r}:=\prod_{t} \operatorname{Sym}\left(\left[r_{t}\right]\right)$ by permutations of the variables, and the homomorphism defining $I_{r}$ is $G_{r}$-equivariant. We want to let some of the $r_{t}$, namely, those with $t$ in a given subset $T \subseteq[m]$, tend to infinity, and conclude that the ideals $I_{r}$ stabilise up to $G_{r}$-symmetry. To put this statement in the context of Chapter 4, we give the $r_{t}$ with $t \notin T$ some fixed values, and take the $r_{t}, t \in T$ all equal, say to $n \in \mathbb{N}$. The corresponding rings and ideals are called $R_{n}, Q_{n}, I_{n}$. We have inclusions $\iota_{n}: R_{n} \rightarrow R_{n+1}$ obtained by inclusion of variables and projections $\pi_{n}: R_{n+1} \rightarrow R_{n}$ obtained by mapping all variables to zero that have at least one $T$-labelled index equal to $n+1$. This maps $I_{n+1}$ into $I_{n}$ because there is a compatible homomorphism $Q_{n+1} \rightarrow Q_{n}$ setting the relevant variables equal to zero. As in Chapter 4 we write $R_{\infty}, I_{\infty}$ for the union of all $R_{n}, I_{n}$.

The group $\operatorname{Sym}(n)^{T}$ acts on $R_{n}, Q_{n}, I_{n}$, but in fact the independent set theorem only needs one copy of $\operatorname{Sym}(n)$ acting diagonally. The additional assumption (*) holds by the same reasoning as in Example 4.2

TheOREM 5.1 (Independent set theorem [HS12].). Suppose that $T \subseteq[m]$ is an independent set in $\Gamma$, i.e., that $T$ intersects any $F \in \Gamma$ in at most one element. Then there exists an $n_{0}$ such that $I_{n}$ is generated by $\operatorname{Sym}(n) I_{n_{0}}$ for all $n \geq n_{0}$.

The condition that $T$ is an independent set cannot simply be dropped. For instance, if $m=3$ and $\Gamma=\{\{1,2\},\{2,3\},\{3,1\}\}$ (the model of no three-way interaction), then if $r_{1}=n$ tends to infinity for fixed $r_{2}, r_{3}$, then the ideal stabilises (see AT03 for the case of $r_{2}=r_{3}=3$ ); but if $r_{1}=r_{2}=n$ both tend to infinity and $r_{1}$ is fixed, say, to 2 , then the ideal does not stabilise DS98. 


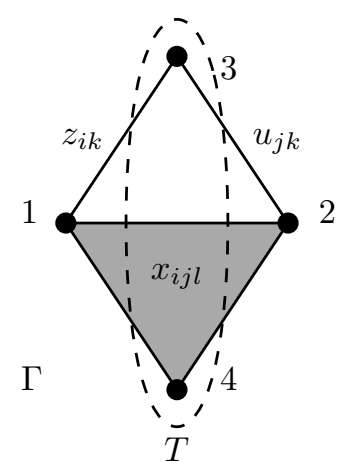

FIGURE 5.1. A hypergraph $\Gamma$ with parameters $x_{i j l}, z_{i k}, u_{j k}$ and independent set $T$.

EXAmple 5.2. As an example take $m=4$ and $\Gamma=\{124,13,23\}$, where 124 is short-hand for $\{1,2,4\}$, etc. We write

$$
y_{i j k l}, x_{i j l}, z_{i k}, u_{j k} \text { instead of } y_{i_{1}, i_{2}, i_{3}, i_{4}}, x_{124,\left(i_{1}, i_{2}, i_{4}\right)}, x_{13,\left(i_{1}, i_{3}\right)}, x_{23,\left(i_{2}, i_{3}\right)},
$$

respectively; see Figure 5.1. The ideal $I_{n}$ is the kernel of the homomorphism sending $y_{i j k l}$ to $x_{i j l} z_{i k} u_{j k}$. Take $T=\{3,4\}$, an independent set in $\Gamma$. The ideal $I_{\infty}$ contains obvious quadratic binomials such as

$$
y_{i j k l} y_{i j k^{\prime} l^{\prime}}-y_{i j k l^{\prime}} y_{i j k^{\prime} l}
$$

with $i \in\left[r_{1}\right], j \in\left[r_{2}\right], k, l, k^{\prime}, l^{\prime} \in \mathbb{N}$. Indeed, the first monomial maps to $x_{i j l} z_{i k} u_{j k}$. $x_{i j l^{\prime}} z_{i k^{\prime}} u_{j k^{\prime}}$, and the second monomial maps to $x_{i j l^{\prime}} z_{i k} u_{j k} \cdot x_{i j l} z_{i k^{\prime}} u_{j k^{\prime}}$, which is the same thing.

These obvious binomials generalise verbatim to the general case, where they read

$$
y_{j k l} y_{j k^{\prime} l^{\prime}}-y_{j k l^{\prime}} y_{j k^{\prime} l}
$$

where now $j$ runs over the finite set $\prod_{t \in[m] \backslash T}\left[r_{t}\right], k, k^{\prime}$ run over $\mathbb{N}^{S}$ and $l, l^{\prime}$ run over $\mathbb{N}^{T \backslash S}$ for some $S$ that runs over all subsets of $T$. Indeed, for any variable $x=x_{F,\left(i_{t}\right)_{t \in F}}$ at most one $t \in F$ lies in $T$. If such a $t$ exists for $x$ and lies in $S$, then whether $x$ appears in the image of a variable $y_{j k l}$ does not depend on the value of $l$. But disregarding that third index, the two monomials above are the same. A similar reasoning for the case where $t \in T \backslash S$ and for the case where $F \cap T=\emptyset$ shows that $x$ has the same exponent in the image of both monomials in the binomial above.

By Proposition 4.1 relating chains to infinite-dimensional varieties, we are done if we can prove that $I_{\infty}$ is generated by $\operatorname{Sym}(\infty) I_{n}$ for some finite $n$. Let $J_{n}, J_{\infty} \subseteq$ $I_{n}, I_{\infty}$, respectively, be the ideals generated by all quadratic binomials as in (1). We claim that $J_{\infty}$ is generated by $\operatorname{Sym}(\infty) J_{2|T|}$. Indeed, for any binomial as above, the set of all indices appearing in $k, l, k^{\prime}, l^{\prime}$ has cardinality $n \leq 2|T|$, and there exists a bijection in $\operatorname{Sym}(\infty)$ mapping its support bijectively onto $[n]$, witnessing that the binomial lies in $\operatorname{Sym}(\infty) J_{2|T|}$. The remainder of the proof consists of showing that the quotient $R_{\infty} / J_{\infty}$ is, in fact, $\operatorname{Sym}(\infty)$-Noetherian. To this end, we introduce a 
new polynomial ring

$$
P:=K\left[y_{j t q}^{\prime} \mid j \in \prod_{t \in[m] \backslash T}\left[r_{t}\right], t \in T, \text { and } q \in \mathbb{N}\right],
$$

where the $y_{j t q}^{\prime}$ are new variables, and consider the subring $R_{\infty}^{\prime}$ of $P$ generated by all monomials $m_{j i}:=\prod_{t \in T} y_{j t i t}^{\prime}$ with $j$ as before and $i \in \mathbb{N}^{T}$. The monomials $m_{j i}$ satisfy the binomial relations (1) (for all splittings of $i$ into two subsequences $k$ and $l$ ), and it is known that these binomials generate the ideal of relations among the $m_{j i}$-indeed, the ring $R_{\infty}^{\prime}$ is the coordinate ring of the Cartesian product of $\prod_{t \in[m] \backslash T} r_{T}$-many copies of the variety of pure $|T|$-dimensional tensors. Thus we have an isomorphism $R_{\infty}^{\prime} \cong R_{\infty} / J_{\infty}$, and we want to show that $R_{\infty}^{\prime}$ is $\operatorname{Sym}(\infty)$ Noetherian. The enveloping polynomial ring $P \supseteq R_{\infty}^{\prime}$ is $\operatorname{Sym}(\infty)$-Noetherian by Theorem 2.3 (only the index $q$ of the variables $y_{j t q}^{\prime}$ is unbounded), but passing to a subring one may, in general, lose Noetherianity. However, let $H$ be the torus in $\left(K^{*}\right)^{T}$ consisting of $T$-tuples of non-zero scalars whose product is 1 . Then the $m_{j i}$ are $H$-invariant, and these monomials generate the ring of $H$-invariant polynomials (if, as we may assume, $K$ is infinite). Hence by Proposition 3.1 we may conclude that $R_{\infty}^{\prime}$ is $\operatorname{Sym}(\infty)$-Noetherian, and this concludes the proof of the independent set theorem. 



\section{CHAPTER 6}

\section{The Gaussian $k$-factor model}

This chapter discusses finiteness results for a model from algebraic statistics known as the Gaussian $k$-factor model. General stabilisation results for this model were first conjectured in DSS07, and for 1 factor established prior to that in dLST95. For 2 factors, a positive-definite variant was established in [DX10, and an ideal-theoretic variant in BD11. The ideal-theoretic version for more factors is open, but the set-theoretic version was established in [Dra10.

The Gaussian $k$-factor model consists of all covariance matrices for a large number $n$ of jointly Gaussian random variables consistent with the hypothesis that those variables can be written as linear combinations of a small number $k$ of hidden factors plus independent, individual noise. Algebraically, let $R_{n}$ be the $K$-algebra of polynomials in variables $y_{i j}=y_{j i}$ with $i, j \in[n]$, and let $P_{k n}$ be the $K$-algebra of polynomials in the variables $x_{i l}, i \in[n], l \in[k]$ and further variables $z_{1}, \ldots, z_{n}$. Let $I_{k n}$ be the kernel of the homomorphism $\phi_{k n}: R_{n} \rightarrow P_{k n}$ that maps $y_{i j}$ to the $(i, j)$-entry of the matrix

$$
x \cdot x^{T}+\operatorname{diag}\left(z_{1}, \ldots, z_{n}\right),
$$

where we interpret $x$ as an $[n] \times[k]$-matrix of variables. Set $S_{k n}:=R_{n} / I_{k n}$; the set $S_{k n}(K) \subseteq K^{\left(\begin{array}{c}n \\ 2\end{array}\right)}$ of $K$-valued points of $S_{k n}$ is (the Zariski closure of) the Gaussian $k$-factor model. Observe that $\phi_{k n}$ is $\operatorname{Sym}([n])$-equivariant, so that $I_{k n}$ is $\operatorname{Sym}([n])$ stable. We are in the setting of Chapter 4, with the map $\pi_{n}: R_{n+1} \rightarrow R_{n}$ mapping $y_{i,(n+1)}$ equal to 0 for all $i$ and the map $\iota_{n}: R_{n} \rightarrow R_{n+1}$ the inclusion. The technical assumption $\left({ }^{*}\right)$ from that chapter holds for the same reason as in Example 4.2 .

TheOREm 6.1. For every fixed $k \in \mathbb{N}$, there exists an $n_{k} \in \mathbb{N}$ such that for all $n \geq n_{k}$ the variety $S_{k n}(K) \subseteq K^{\left(\begin{array}{c}n+1 \\ 2\end{array}\right)}$ is cut out set-theoretically by the polynomials in $\operatorname{Sym}(n) I_{n_{k}}$.

Proof. By Proposition 4.1 and the discussion following its proof we need only prove that $S_{k \infty}(K)$ is the zero set of $\operatorname{Sym}(\infty) I_{n_{k}}$, for suitable $n_{k}$. Let $J_{k \infty}$ denote the ideal generated by all $(k+1) \times(k+1)$-minors of the symmetric $\mathbb{N} \times \mathbb{N}$-matrix $y$ that do not involve diagonal entries of $y$, and set $S_{k \infty}^{\prime}:=R_{\infty} / J_{k \infty}$. Then surely $J_{k \infty}$ is contained in $I_{k \infty}$, so that, dually, $S_{k \infty}^{\prime}(K)$ contains $S_{k \infty}(K)$.

We claim that $S_{k \infty}^{\prime}(K)$ is a $\operatorname{Sym}(\infty)$-Noetherian topological space, and to prove this claim we proceed by induction. For $k=0$ the equations in $J_{k \infty}=J_{0 \infty}$ force all off-diagonal entries of the matrix $y$ to be zero, so that $S_{0 \infty}^{\prime}(K)$ is just the set of $K$ points of $K\left[y_{11}, y_{22}, \ldots\right]$, with $\operatorname{Sym}(\infty)$ permuting the coordinates. The latter ring is $\operatorname{Sym}(\infty)$-Noetherian by Theorem 2.3. and hence its topological space of $K$-points is certainly $\operatorname{Sym}(\infty)$-Noetherian.

Next, assume that $S_{k-1, \infty}^{\prime}(K)$ is $\operatorname{Sym}(\infty)$-Noetherian, and note that $S_{k-1, \infty}^{\prime}(K)$ is a (closed) subset of $S_{k, \infty}^{\prime}(K)$. On any point outside this closed subset at least one 


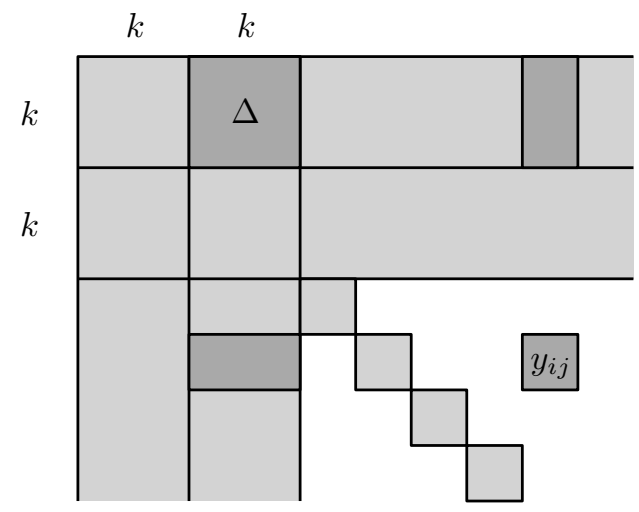

Figure 6.1. The set $T$ labelling symmetric matrix entries (in light gray), and the $(k+1) \times(k+1)$-determinant expressing $y_{i j}$ as a rational function in the $T$-labelled variables (in dark gray).

of the $k \times k$-determinants in $J_{k-1, \infty}$ is non-zero. Up to signs, these determinants form a single orbit under $\operatorname{Sym}(\infty)$, so if we set $\Delta:=\operatorname{det} y[[k],[2 k] \backslash[k]]$, then we have

$$
\begin{aligned}
S_{k, \infty}^{\prime}(K) & =S_{k-1, \infty}^{\prime}(K) \cup Z \operatorname{Sym}(\infty), \text { where } \\
Z & =\left\{y \in S_{k \infty}^{\prime}(K) \mid \Delta \neq 0\right\} .
\end{aligned}
$$

The union of two $\operatorname{Sym}(\infty)$-Noetherian topological spaces is $\operatorname{Sym}(\infty)$-Noetherian, so it suffices to prove that $Z \operatorname{Sym}(\infty)$ is $\operatorname{Sym}(\infty)$-Noetherian. Observe that $Z$ itself is stable under the subgroup $H:=\left\{\pi \in \operatorname{Sym}(\infty)|\pi|_{[2 k]}=\left.1\right|_{[2 k]}\right\}$. Hence by Proposition 3.5 it suffices to prove that $Z$ is $H$-Noetherian. To this end, define $T \subseteq \mathbb{N} \times \mathbb{N}$ by

$$
T:=\{(i, j) \in \mathbb{N} \times \mathbb{N} \mid i=j \text { or }(i<j \text { and } i \in[2 k])\} .
$$

Let $Q$ be the open subset of $K^{T}$ where the $[k] \times([2 k] \backslash[k])$-submatrix has non-zero determinant. The coordinate ring of $Q$ is $H$-Noetherian by Theorem 2.3 and the fact that adding finitely many $H$-fixed variables preserves $H$-Noetherianity. As a consequence, $Q$ is an $H$-Noetherian space. We claim that the projection $\operatorname{pr}: Z \rightarrow Q$ that maps a matrix $y$ to its $T$-labelled entries is a closed, $H$-equivariant embedding. Equivariance is immediate. To see that pr is injective observe that, for $y \in Z$, any matrix entry $y_{i j}$ with $i<j$ (since we work with symmetric matrices) and $i \notin[2 k]$ satisfies an equation

$$
0=\operatorname{det} y[[k] \cup\{i\},([2 k] \backslash[k]) \cup\{j\}]=\Delta \cdot y_{i j}-E,
$$

where $E$ is an expression involving only variables in $T$. Since $\Delta$ is non-zero, we find that $y_{i j}$ is determined by $\operatorname{pr}(y)$. This shows injectivity. That $\operatorname{pr}: Z \rightarrow Q$ is, in fact, a closed embedding follows by showing that the dual map $K[Q] \rightarrow K[Z]$ is surjective: the regular function $\frac{E}{\operatorname{det} \Delta}$ maps onto $\left.y_{i j}\right|_{Z}$. Since $Q$ is $H$-Noetherian, so is $Z$, and as mentioned before this concludes the induction step.

As $S_{k, \infty}^{\prime}(K)$ is Noetherian, we find that in particular, the Zariski closure $S_{k, \infty}(K)$ is cut out from $S_{k, \infty}^{\prime}(K)$ by finitely many $\operatorname{Sym}(\mathbb{N})$-orbits of equations. Representatives of these orbits already lie in $S_{k, n_{k}}^{\prime}(K)$ for suitable $n_{k}$. 


\section{CHAPTER 7}

\section{Tensors and $\Delta$-varieties}

This chapter deals with finiteness results for a wide class of varieties of tensors, introduced by Snowden $\mathbf{S n o 1 3}$ and called $\Delta$-varieties. The proof of this chapter's theorem is more involved than earlier proofs, and we have therefore decided to break the chapter up into more digestible sections.

$\Delta$-varieties. We work over a ground field $K$, which we assume to be infinite to avoid anomalies with the Zariski topology. For any tuple $\left(V_{1}, \ldots, V_{n}\right)$ of finite-dimensional vector spaces over $K$, we write $\mathbf{V}\left(V_{1}, \ldots, V_{n}\right)$ for the tensor product $V_{1}^{*} \otimes \cdots \otimes V_{n}^{*}$. These spaces have three types of interesting maps between them. First, given linear maps $f_{i}: V_{i} \rightarrow W_{i}$ there is a natural linear map $\mathbf{V}\left(W_{1}, \ldots, W_{n}\right) \rightarrow \mathbf{V}\left(V_{1}, \ldots, V_{n}\right)$, namely, the tensor product $\otimes_{i} f_{i}^{*}$ of the dual maps $f_{i}^{*}$. Second, given any $\sigma \in \operatorname{Sym}([n])$, there is a canonical map $\sigma: \mathbf{V}\left(V_{\sigma(1)}, \ldots, V_{\sigma(n)}\right) \rightarrow \mathbf{V}\left(V_{1}, \ldots, V_{n}\right)$. Third, there is a canonical flattening map $\mathbf{V}\left(V_{1}, \ldots, V_{n}, V_{n+1}\right) \rightarrow \mathbf{V}\left(V_{1}, \ldots, V_{n} \otimes V_{n+1}\right)$, which is called like this because, in coordinates, it takes an $(n+1)$-way table of numbers and transforms it into an $n$-way table; see Figure 7.1

A $\Delta$-variety is not a single variety, but rather a rule $\mathbf{X}$ that takes as input a finite sequence $\left(V_{j}\right)_{j \in[n]}$ of finite-dimensional vector spaces over $K$, and assigns to it a subvariety $\mathbf{X}\left(V_{1}, \ldots, V_{n}\right)$ of $\mathbf{V}\left(V_{1}, \ldots, V_{n}\right)$. To be a $\Delta$-variety, each of the three types of maps above must preserve $\mathbf{X}$, i.e., $\otimes_{i} f_{i}^{*}$ must map $\mathbf{X}\left(W_{1}, \ldots, W_{n}\right)$ into $\mathbf{X}\left(V_{1}, \ldots, V_{n}\right)$, and $\sigma$ must map $\mathbf{X}\left(V_{\sigma(1)}, \ldots, V_{\sigma(n)}\right)$ into $\mathbf{X}\left(V_{1}, \ldots, V_{n}\right)$, and the flattening map must map $\mathbf{X}\left(V_{1}, \ldots, V_{n+1}\right)$ into $\mathbf{X}\left(V_{1}, \ldots, V_{n} \otimes V_{n+1}\right)$.

The $\Delta$-varieties that we shall study will have a fourth, additional property, namely, that the inverse to the isomorphism $\mathbf{V}\left(V_{1}, \ldots, V_{n}, K\right) \rightarrow \mathbf{V}\left(V_{1}, \ldots, V_{n} \otimes\right.$ $K)=\mathbf{V}\left(V_{1}, \ldots, V_{n}\right)$ maps $\mathbf{X}\left(V_{1}, \ldots, V_{n}\right)$ into $\mathbf{X}\left(V_{1}, \ldots, V_{n}, K\right)$; we call such $\Delta$ varieties good. Taking any linear function $f$ from an additional vector space $V_{n+1}$ to $K$ we then find that $\mathbf{X}\left(V_{1}, \ldots, V_{n}\right) \otimes f$, being the image of $\mathbf{X}\left(V_{1}, \ldots, V_{n}\right)$ under the map above followed by $1_{V_{1}}^{*} \otimes \cdots \otimes 1_{V_{n}}^{*} \otimes f^{*}$, is contained in $\mathbf{X}\left(V_{1}, \ldots, V_{n}, V_{n+1}\right)$.
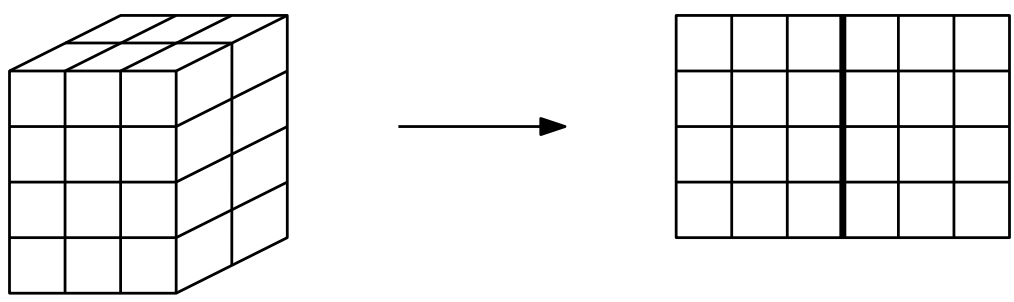

FiguRE 7.1. Flattening an element of $K^{4} \otimes K^{2} \otimes K^{3}$ to an element of $K^{4} \otimes\left(K^{2} \otimes K^{3}\right)$. 
A (boring) example of a $\Delta$-variety that is not good is that for which $\mathbf{X}\left(V_{1}, \ldots, V_{n}\right)$ equals $\mathbf{V}\left(V_{1}, \ldots, V_{n}\right)$ if $n<10$ and the empty set otherwise.

A typical example of a good $\Delta$-variety is Seg, the cone over Segre, which maps a tuple of vector spaces to the variety of pure tensors $v_{1} \otimes \cdots \otimes v_{n}$ in the tensor product of the duals. In fact, any non-empty good $\Delta$-variety contains Seg-but the class of good $\Delta$-varieties is much larger. For instance, it is closed under taking joins: if $\mathbf{X}$ and $\mathbf{Y}$ are $\Delta$-varieties, then the rule $\mathbf{X}+\mathbf{Y}$ that assigns to $\left(V_{i}\right)_{i \in[n]}$ the Zariski closure of $\mathbf{X}\left(V_{1}, \ldots, V_{n}\right)+\mathbf{Y}\left(V_{1}, \ldots, V_{n}\right)$ is also a $\Delta$-variety, and good if both $\mathbf{X}$ and $\mathbf{Y}$ are. Similarly, (good) $\Delta$-varieties are closed under taking tangential varieties, unions, and intersections.

Given some equations for an instance of a $\Delta$-variety $\mathbf{X}$, one obtains equations for other instances of $\mathbf{X}$ by pulling back along sequences of the maps appearing in the definition of a $\Delta$-variety. For instance, start with the $2 \times 2$-determinant defining $\operatorname{Seg}\left(K^{2}, K^{2}\right)$ inside $\mathbf{V}\left(K^{2}, K^{2}\right)$. Then we obtain generators for the ideal of $\operatorname{Seg}\left(K^{m}, K^{n}\right)$ by pulling the determinant back along duals of linear maps $f_{1}$ : $K^{2} \rightarrow K^{m}$ and $f_{2}: K^{2} \rightarrow K^{n}$ And then, using the remaining two axioms, we also find equations for the variety of pure tensors in, say, $\mathbf{V}\left(K^{2}, K^{2}, K^{2}, K^{2}\right)$ through the flattening maps into $\mathbf{V}\left(K^{2} \otimes K^{2}, K^{2} \otimes K^{2}\right) \cong \mathbf{V}\left(K^{4}, K^{4}\right), \mathbf{V}\left(K^{2}, K^{2} \otimes K^{2} \otimes K^{2}\right) \cong$ $\mathbf{V}\left(K^{2}, K^{8}\right)$, etc. Indeed, one readily shows that one obtains generators of the ideals of all instances of Seg in this manner. The result in this chapter is that a similar result holds for any sufficiently small good $\Delta$-variety, at least at a topological level.

TheOREM 7.1. Let $\mathbf{X}$ be a good $\Delta$-variety which is bounded in the sense that there exist finite-dimensional vector spaces $W_{1}, W_{2}$ such that $\mathbf{X}\left(W_{1}, W_{2}\right)$ is not all of $\mathbf{V}\left(W_{1}, W_{2}\right)$. Then there exist an $n_{\mathbf{X}} \in \mathbb{N}$ and vector spaces $U_{1}, \ldots, U_{n_{\mathbf{X}}}$ such that $\mathbf{X}$ equals the inclusion-wise largest $\Delta$-variety $\mathbf{Y}$ with $\mathbf{Y}\left(U_{1}, \ldots, U_{n_{\mathbf{X}}}\right)=$ $\mathbf{X}\left(U_{1}, \ldots, U_{n_{\mathbf{X}}}\right)$.

This means, in more concrete terms, that the equations for $\mathbf{X}\left(U_{1}, \ldots, U_{n_{\mathbf{X}}}\right)$, pulled back along all four types of linear maps from the definition of a good $\Delta$ variety, yield equations that cut out all instances of $\mathbf{X}$ from their ambient spaces. In particular, there is a universal degree bound, depending only on $\mathbf{X}$ but not on $n$ or $V_{1}, \ldots, V_{n}$, on equations needed to define $\mathbf{X}\left(V_{1}, \ldots, V_{n}\right)$ set-theoretically within $\mathbf{V}\left(V_{1}, \ldots, V_{n}\right)$.

Since $\operatorname{GL}\left(W_{1}\right) \times \operatorname{GL}\left(W_{2}\right)$ acts with a dense orbit on $\mathbf{V}\left(W_{1}, W_{2}\right)$-namely, the two-tensors (or matrices) of full rank - the boundedness condition on $X$ implies that all two-tensors in instances of the form $\mathbf{X}\left(V_{1}, V_{2}\right)$ have uniformly bounded rank. This readily implies that the boundedness condition on $\mathbf{X}$ is also preserved under joins (by adding the rank bounds), tangential varieties, intersections, and unions, so that the theorem applies to a wide class of $\Delta$-varieties of interest in applications.

Related literature. The boundedness condition on $\Delta$-varieties was first formulated, at an ideal-theoretic level, in [Sno13. There it is conjectured that a generalisation of Theorem 7.1 should hold, for bounded $\Delta$-varieties, on the idealtheoretic level; and not only for equations of instances of $\mathbf{X}$, but also for their $q$ syzygies for any fixed $q \geq 1$. This general statement is proved for Seg in Sno13. The special case where $q=1$, i.e., finiteness of equations, is known to hold for the

\footnotetext{
${ }^{1}$ Snowden chose the notion of $\Delta$-varieties contravariant in the linear maps $f_{i}$ so as to make defining ideals and more general $\Delta$-modules [Sno13] depend covariantly on them.
} 
tangential variety to Seg [OR11, confirming a conjecture from [LW07] and for the variety $\mathbf{S e g}+\mathbf{S e g}=2 \mathbf{S e g}$ of tensors of border rank at most 2 [Rai12], confirming the GSS-conjecture from GSS05 (a set-theoretic version of which was first proved in LM04). The set-theoretic theorem above was first proved in DK13. for $k \mathbf{S e g}$, i.e., for any fixed secant variety of $\mathbf{S e g}$; and a discussion with Snowden led to the insight that our proof generalises to bounded, good, $\Delta$-varieties as in the theorem. Further recent keywords closely related to the topic of this chapter are $\mathrm{GL}_{\infty}$-algebras [SS12a], twisted commutative algebras [SS12b], FI-modules CEF12, and cactus varieties BB13.

From a $\Delta$-variety to an infinite-dimensional variety. We prove the theorem by embedding all relevant instances of $\mathbf{X}$ into a single, infinite-dimensional variety given by determinantal equations, and showing that this variety is Noetherian up to symmetry preserving $\mathbf{X}$. By the boundedness assumption, we can choose a number $p$ that is strictly greater than the ranks of all two-tensors in instances $\mathbf{X}\left(V_{1}, V_{2}\right)$, independently of $V_{1}$ and $V_{2}$. Set $V:=K^{[p]}$ and $X_{n}:=\mathbf{X}(V, \ldots, V) \subseteq$ $\mathbf{V}_{n}:=\mathbf{V}(V, \ldots, V)$, where the number of $V$ s equals $n$. We first argue that the equations for all (infinitely many) $X_{n}, n \in \mathbb{N}$ pull back to equations defining all instances of $\mathbf{X}$.

Indeed, let $V_{1}, \ldots, V_{n}$ be vector spaces, and let $\omega \in \mathbf{V}\left(V_{1}, \ldots, V_{n}\right)$ be a tensor. Then we claim that $\omega$ lies in $\mathbf{X}\left(V_{1}, \ldots, V_{n}\right)$ if and only if for all linear maps $f_{i}$ : $V \rightarrow V_{i}$ the image of $\omega$ under $\otimes_{i \in[n]} f_{i}^{*}$ lies in $X_{n}$. The "only if" claim follows from the first axiom for $\Delta$-varieties. For the "if" claim, note that if $\left(\otimes_{i \in[n]} f_{i}^{*}\right) \omega$ lies in $X_{n}$ for all tuples of $f_{i}$, then for each $j \in[n]$, the linear map that $\omega$ induces from $\bigotimes_{i \neq j} V_{i}$ into $V_{j}^{*}$, being a flattening of $\omega$ in $\mathbf{V}\left(\bigotimes_{i \neq j} V_{i}, V_{j}\right)$, has image $U_{j} \subseteq V_{j}^{*}$ of dimension strictly smaller than $p$. Now take $f_{j}: V \rightarrow V_{j}$ such that $f_{j}^{*}$ restricts to an injection $U_{j} \rightarrow V^{*}$, and let $g_{j}: V_{j} \rightarrow V$ be such that $g_{j}^{*} \circ f_{j}^{*}$ restricts to the identity on $U_{j}$. Then the tensor $\omega^{\prime}:=\otimes_{j} f_{j}^{*} \omega$ lies in $X_{n}=\mathbf{X}(V, \ldots, V)$ by assumption. But then, by the first axiom, the tensor $\otimes_{j} g_{j}^{*} \omega^{\prime}=\omega$ lies in $\mathbf{X}\left(V_{1}, \ldots, V_{n}\right)$, as claimed. This argument actually also works ideal-theoretically; only later shall we need to work purely topologically.

We now cast the chain of varieties $\left(X_{n}\right)_{n \in \mathbb{N}}$ into the framework of Chapter 4 To this end, let $R_{n}$ denote the symmetric $K$-algebra generated by $V^{\otimes[n]}$, which is the coordinate ring of $\mathbf{V}_{n}$. Pick a non-zero element $x_{0} \in V$ and let $\iota_{n}: R_{n} \rightarrow$ $R_{n+1}$ be the homomorphism of $K$-algebras determined by the linear map $V^{\otimes[n]} \rightarrow$ $V^{\otimes[n+1]}, x \mapsto x \otimes x_{0}$. The group $\operatorname{GL}(V)^{[n]}$ acts on $V^{\otimes[n]}$ in the natural manner, and this extends to an action by algebra automorphisms on $R_{n}$. Similarly, the group $\operatorname{Sym}([n])$ acts on $V^{\otimes[n]}$ by permuting tensor factors. The embedding $\iota_{n}$ is equivariant for the group $G_{n}:=\operatorname{Sym}([n]) \ltimes \operatorname{GL}(V)^{[n]}$ if we embed $\operatorname{Sym}([n])$ into $\operatorname{Sym}([n+1])$ by fixing $n$ and $\operatorname{GL}(V)^{[n]}$ into $\operatorname{GL}(V)^{[n+1]}$ by adding $1_{V}$ in the last component.

The linear map $\iota^{*}: \mathbf{V}_{n+1} \rightarrow \mathbf{V}_{n}$ maps $X_{n+1}$ into $X_{n}$, and $X_{n}$ is preserved by $G_{n}$. Letting $S_{n}$ be the coordinate ring of $R_{n}$, we have all the arrows in the diagram of Figure 4.1 except for the arrows to the right. To obtain these, we use that $\mathbf{X}$ is good, as follows. Given any $e_{0} \in V^{*}$ such that $e_{0}\left(x_{0}\right)=1$, the map $\pi_{n}^{*}: \mathbf{V}_{n} \rightarrow \mathbf{V}_{n+1}, \omega \mapsto \omega \otimes e_{0}$ maps $X_{n}$ into $X_{n+1}$. The dual to this linear map, extended to an algebra homomorphism, is the required map $\pi: R_{n+1} \rightarrow R_{n}$. This completes the diagram. The technical condition $*$ from page 21 is also satisfied, i.e., for all indices $l, i_{0}, i_{1}$ with $l \geq i_{0}, i_{1}$ and for all $g \in G_{l}$ there exist an index 
$j \leq i_{0}, i_{1}$ and group elements $g_{0} \in G_{i_{0}}, g_{1} \in G_{i_{1}}$ such that

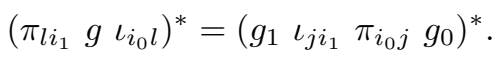

Indeed, the left-hand side is the composition of the map $\mathbf{V}_{i_{1}} \rightarrow \mathbf{V}_{l}$ tensoring with $e_{0}^{\otimes l-i_{1}}$, followed by $g$, followed by the map $\mathbf{V}_{l} \rightarrow \mathbf{V}_{i_{0}}$ contracting with $x_{0}^{\otimes l-i_{0}}$ in the last $l-i_{0}$ factors. Let $i_{1}-j$ be the number of factors $V^{*}$ in $\mathbf{V}_{i_{1}}$ that are moved, by $g$, into the last $l-i_{0}$ positions and hence end up being contracted in the last step. This means that $j \leq i_{0}, i_{1}$ is the number of factors $V^{*}$ in $\mathbf{V}_{i_{1}}$ that are not contracted. Hence the composition can also be obtained by first applying a $g_{1} \in G_{i_{1}}$, ensuring that the $i_{1}-j$ factors $V^{*}$ in $\mathbf{V}_{i_{1}}$ that need to be contracted are in the last $i_{1}-j$ positions; then contracting by a pure tensor in those positions, which for suitable choice of $g_{1}$ may be chosen $x_{0}^{\otimes i_{1}-j}$; then tensoring with $i_{0}-j$ copies of $e_{0}$; and finally applying a suitable element $g_{0} \in G_{i_{0}}$.

The upshot of this is that if we can prove that the projective limit $X_{\infty}:=$ $\lim _{\leftarrow n} X_{n}$ is defined by finitely many $G_{\infty}:=\bigcup_{n} G_{n}$-orbits of equations within $\mathbf{V}_{\infty}:=\lim _{\leftarrow n} \mathbf{V}_{n}$, then there exists an $n_{X}$ such that for all $n \geq n_{X}$ the variety $X_{n}$ is defined by the $G_{n}$-orbits of equations for $X_{n_{X}}$. This implies the theorem.

Flattening varieties. To prove this finiteness result, then, we show that $X_{\infty}$ is contained in a $G_{\infty}$-Noetherian subvariety $Y_{\infty}$ of $\mathbf{V}_{\infty}$, which we call a flattening variety, and that $Y_{\infty}$ itself is defined by finitely many $G_{\infty}$-orbits of equations. To define $Y_{\infty}$, let $\mathbf{Y}^{(k)}$ denote the largest $\Delta$-variety for which $\mathbf{Y}^{(k)}\left(V_{1}, V_{2}\right)$ consists of two-tensors of rank at most $k$. Then $\mathbf{Y}^{(k)}\left(V_{1}, \ldots, V_{n}\right)$ is defined by the vanishing of all $(k+1) \times(k+1)$-minors of the flattenings $\mathbf{V}\left(V_{1}, \ldots, V_{n}\right) \rightarrow \mathbf{V}\left(\bigotimes_{i \in A} V_{i}, \bigotimes_{i \in B} V_{i}\right)$ for all partitions of $[n]$ into disjoint subsets $A$ and $B$. Set $Y_{n}^{(k)}:=\mathbf{Y}^{(k)}(V, \ldots, V) \subseteq$ $\mathbf{V}_{n}$, and $Y_{\infty}^{(k)}:=\lim _{\leftarrow n} Y_{n}^{(k)} \subseteq \lim _{\leftarrow n} \mathbf{V}_{n}$. By the boundedness assumption on $\mathbf{X}$, $Y_{\infty}^{(p-1)}$ contains $X_{\infty}$.

We first prove that each $Y_{\infty}^{(k)}, k \in \mathbb{N}$ is defined by finitely many $G_{\infty}$-orbits of equations. Unwinding the definitions, this statement boils down to the statement that if $\omega \in \mathbf{V}_{n}$ with $n \gg 0$ does not lie in $Y_{n}^{(k)}$, then there exists an $i \in[n]$ and an $x \in V$ such that contracting $\omega$ with $x$ in the $i$-th position yields a tensor $\omega^{\prime} \in \mathbf{V}_{n-1}$ that does not lie in $Y_{n-1}^{(k)}$. In fact, we shall see that $n>2 k$ suffices. The condition that $\omega$ does not lie in $Y_{n}^{(k)}$ means that there is a partition $[n]=A \cup B$ such that $\omega$, regarded as a linear map $V^{\otimes A} \rightarrow\left(V^{*}\right)^{\otimes B}$, has rank strictly larger than $k$. Using that $n>2 k$ and after swapping $A$ and $B$ if necessary we may assume that $|B|>k$.

Let $U \subseteq\left(V^{*}\right)^{\otimes B}$ be a $(k+1)$-dimensional subspace of the image of this linear map $\omega$. We claim that since $|B|$ is larger than $k$, there exists a position $i \in B$ and an $x \in V$ such that the image of $U$ under contraction with $x$ in the $i$-th position still has dimension $k+1$. Indeed, otherwise $U$ would be a point in the projective variety

$$
\begin{aligned}
Q:=\left\{W \in \operatorname{Gr}_{k+1}\left(V^{*}\right)^{\otimes B} \mid\right. & \text { contracting } U \text { with any } x \text { in any position } \\
& \text { decreases the dimension }\} .
\end{aligned}
$$

We claim that this variety is empty. To prove this, extend the distinguished vector $x_{0} \in V$ from the definition of $\mathbf{V}_{\infty}$ to a basis $x_{0}, \ldots, x_{p-1}$, where the distinguished 
$e_{0} \in V^{*}$ vanishes on $x_{1}, \ldots, x_{p-1}$. Then the basis of $V^{*}$ dual to $x_{0}, \ldots, x_{p-1}$ starts with $e_{0}$; denote it $e_{0}, \ldots, e_{p-1}{ }^{2}$

If $Q$ is not empty, then by Borel's fixed point theorem Bor91 $Q$ contains a $T^{B}$-fixed point $W$, where $T$ is the maximal torus in $\mathrm{GL}(V)$ consisting of invertible linear maps whose matrices with respect to $x_{0}, \ldots, x_{p-1}$ are diagonal. This means that $W$ has a basis of common eigenvectors $e_{\alpha}:=\otimes_{i \in B} e_{\alpha_{i}}$, where $\alpha$ runs through some set $J \subseteq\{0, \ldots, p-1\}^{B}$ of cardinality $k+1$. Think of the $\alpha \in J$ as $B$-labelled words over the alphabet $\{0, \ldots, p-1\}$. Contracting $e_{\alpha}$ with $x_{0}+x_{1}+\ldots+x_{p-1}$ at position $i \in B$ yields $e_{\alpha^{\prime}}$, where $\alpha^{\prime}$ is the word obtained from $\alpha$ by deleting the $i$-th letter. By assumption, the resulting words $e_{\alpha^{\prime}}, \alpha \in J$ are linearly dependent, which means that at least two of them must coincide.

Summing up, $J$ consists of $k+1$ distinct words of length $|B| \geq k+1$ with the property that for each $i \in B$ the collection $J$ contains two words that differ only at position $i$. By induction on $k$ we show that this is impossible, i.e., that for $k+1$ distinct words of length $\geq k+1$ over any alphabet there exist $k$ positions restricted to which all words are distinct. For $k=0$ this is immediate: restricting a single word to zero positions yields a single (empty) word. Assume that it is true for $k-1$, and consider $k+1$ words of length $\geq k+1$. Set one word $\alpha$ apart. Then there exist $k-1$ positions restricted to which the remaining $k$ words are distinct. Restricted to those $k-1$ positions $\alpha$ equals at most one word $\alpha^{\prime}$ of the remaining words. So by adding to the $k-1$ positions a position where $\alpha$ and $\alpha^{\prime}$ differ we obtain $k$ positions restricted to which all words are distinct.

This contradiction shows that there exists an $i \in B$ and an $x \in V$ such that the contraction of $U$ with $x$ at position $i$ still has dimension $k+1$. As a consequence, contracting $\omega$ with $x$ at position $i$ yields a tensor outside $Y_{n-1}^{(k)}$, as claimed. Thus $Y_{\infty}^{(k)}$ is defined by finitely many $G_{\infty}$-orbits of equations.

The variety $Y_{\infty}^{(k)}$ is defined by the vanishing of $(k+1) \times(k+1)$-determinants. For what follows, it will be convenient to understand these explicitly in terms of coordinates. The basis $x_{0}, \ldots, x_{p-1}$ gives rise to a basis $x_{w}, w \in\{0, \ldots, p-1\}^{[n]}$ of $V^{\otimes[n]}$. The ring $R_{n}$ is the polynomial ring in these variables. Under the embedding $\iota_{n}: R_{n} \rightarrow R_{n+1}$ the variable $x_{w}$ is mapped to $x_{w 0}$. Hence $R_{\infty}$ is the polynomial ring in variables $x_{w}$ where $w$ runs over all infinite words in $\{0, \ldots, p-1\}^{\mathbb{N}}$ of finite support $\operatorname{supp}(w):=\left\{j \in \mathbb{N} \mid w_{j} \neq 0\right\}$; let us call these finitary words. In these coordinates, a determinantal equation for $Y_{\infty}^{(k)}$ looks as follows. Fix $k+1$ finitary words $w_{i}, i \in[k+1]$ and $k+1$ further finitary words $w_{j}^{\prime}, j \in[k+1]$ with the requirement that $\operatorname{supp}\left(w_{i}\right) \cap \operatorname{supp}\left(w_{j}^{\prime}\right)=\emptyset$ for all $i, j$. Then form the square matrix

$$
x\left[\left(w_{i}\right)_{i},\left(w_{j}^{\prime}\right)_{j}\right]:=\left(x_{w_{i}+w_{j}^{\prime}}\right)_{i, j \in[k+1]}
$$

and its determinant

$$
\Delta\left[\left(w_{i}\right)_{i},\left(w_{j}^{\prime}\right)_{j}\right]:=\operatorname{det} x\left[\left(w_{i}\right)_{i},\left(w_{j}^{\prime}\right)_{j}\right] .
$$

All determinants defining $Y_{\infty}^{(k)}$ have this form $3^{3}$

\footnotetext{
${ }^{2}$ The reason for labelling with $\{0, \ldots, p-1\}$ rather than $[p]$ will become apparent soon.

${ }^{3}$ The convenient fact that the sum of two finitary words is again finitary explains our choice of labelling $x_{0}, \ldots, x_{p-1}$.
} 
Noetherianity of flattening varieties. Using this explicit understanding of the defining equations for $Y_{\infty}^{(k)}$, we prove that $Y_{\infty}^{(k)}$, with its Zariski-topology, is $G_{\infty}$-Noetherian. The proof is similar to that in Chapter 6 for the Gaussian $k$ factor model. In particular, we proceed by induction on $k$. For $k=0$ the variety $Y_{\infty}^{(0)}$ consists of a single point, namely, 0 , and is certainly Noetherian. Now assume that $Y_{\infty}^{(k-1)}$ is $G_{\infty}$-Noetherian. By the discussion of flattening varieties, $Y_{\infty}^{(k-1)}$ is defined by the orbits of finitely many $k \times k$-determinants of flattenings, say $q$ of them. Let $\Delta_{a}, a \in[q]$ be those determinants. Then we may write

$$
\begin{aligned}
Y_{\infty}^{(k)} & :=Y_{\infty}^{(k-1)} \cup \bigcup_{a \in[q]} Z_{a} G_{\infty}, \text { where } \\
Z_{a} & :=\left\{\omega \in Y_{\infty}^{(k)} \mid \Delta_{a}(\omega) \neq 0\right\} .
\end{aligned}
$$

As in the case of the $k$-factor model, it suffices to show that $Z_{a}$ is Noetherian under a suitable subgroup of $G_{\infty}$ stabilising it. To this end, write $\Delta_{a}=\Delta\left[\left(w_{i}\right)_{i},\left(w_{j}^{\prime}\right)_{j}\right]$ for $k$ finitary words $w_{i}$ and $k$ finitary words $w_{j}^{\prime}$ with $\operatorname{supp}\left(w_{i}\right) \cap \operatorname{supp}\left(w_{j}^{\prime}\right)=\emptyset$ for all $i, j$. Set $n:=\max \left(\bigcup_{i} \operatorname{supp}\left(w_{i}\right) \cup \bigcup_{j} \operatorname{supp}\left(w_{j}^{\prime}\right)\right)$ and observe that $\Delta_{a}$ is fixed by $H:=\left\{\pi \in \operatorname{Sym}(\infty)|\pi|_{[n]}=1_{[n]}\right\}$, and hence $Z_{a}$ is stabilised by $H$. We claim that $Z_{a}$ is $H$-Noetherian. To prove this, let $J$ be the set of finitary words $w$ with $|\operatorname{supp}(w) \backslash[n]| \leq 1$. In particular, all variables appearing in $\Delta_{a}$ are in $J$. Let $Q$ be the open subset of $K^{J}$ where $\Delta_{a}$ is non-zero. By Theorem 2.3 and the fact that adding finitely many $H$-fixed variables to an $H$-Noetherian ring preserves $H$-Noetherianity, the coordinate ring of $Q$ is $H$-Noetherian-here the crucial point is that "only one index runs off to infinity". We claim that the projection pr: $Z_{a} \rightarrow Q$ mapping a point to its coordinates labelled by $J$ is an $H$-equivariant, closed embedding. Equivariance is immediate. To see that pr is injective, we prove that on $Z_{a}$ any variable $x_{w}$ has an expression in terms of the variables labelled by $J$. We proceed by induction on the cardinality of $\operatorname{supp}(w) \backslash[n]$. For cardinality 0 and 1 the word $w$ lies in $J$ and we are done. So assume that the cardinality is at least 2 and that the statement is true for all smaller cardinalities. Then we can split $w$ as $u+u^{\prime}$ where $\operatorname{supp}(u) \cap \operatorname{supp}\left(u^{\prime}\right), \operatorname{supp}(u) \cap \operatorname{supp}\left(w_{j}^{\prime}\right)$, and $\operatorname{supp}\left(w_{i}\right) \cap \operatorname{supp}\left(u^{\prime}\right)$ are all empty and where both $\operatorname{supp}(u)$ and $\operatorname{supp}\left(u^{\prime}\right)$ contain at least one element of $\mathbb{N} \backslash[n]$. Then on $Z_{a}$ we have

$$
0=\Delta\left[\left(w_{1}, \ldots, w_{k}, u\right),\left(w_{1}^{\prime}, \ldots, w_{k}^{\prime}, u^{\prime}\right)\right]=\Delta_{a} \cdot x_{u+u^{\prime}}-E
$$

where $E$ is an expression involving only variables whose supports contain fewer elements of $\mathbb{N} \backslash[n]$ than $\operatorname{supp}(w)$ does. By the induction hypothesis, these may be expressed in the variables labelled by $J$, and as $\Delta_{a}$ is non-zero on $Z_{a}$, so can $x_{u+u^{\prime}}=x_{w}$. To show that pr is a closed embedding we note that the map $K[Q] \rightarrow$ $K[Z]$ is surjective: there is an expression for $\left.E\right|_{Z}$ involving only $J$-labelled variables, and dividing by $\Delta_{a}$ yields such an expression for $x_{w}$.

We conclude that $Z$ has the topology of a closed $H$-stable subspace of $K^{Q}$ and is hence $H$-Noetherian. By Proposition 3.5 and the fact that finite unions of equivariantly Noetherian spaces are equivariantly Noetherian, we find that $Y_{\infty}^{(k)}$ is $G_{\infty}$-Noetherian. This concludes the proof of the theorem.

An important final remark is in order here: our proof of the theorem shows that $X_{\infty}$ is defined by finitely many $G_{\infty}$-orbits of equations, which is stronger than the theorem claims. In particular, this stronger statement can be used to show that 
for each fixed $\Delta$-variety there is a polynomial-time membership test. On the other hand, it is typically not true that the ideal of $X_{\infty}$ is generated by finitely many $G_{\infty^{-}}$ orbits of polynomials; indeed, this statement is already false for the cone over Segre. How to reconcile this with the aforementioned conjecture Sno13 that an idealtheoretic version of theorem should hold? Well, by pulling back equations along elements of $G_{\infty}$, we are implicitly pulling back equations along tensor products of linear maps, and along permutations of tensor factors, and along contractions, and along tensoring with $e_{0}$, but not along flattening maps (though we did use, in the proof, that $\mathbf{X}$ was closed under flattening). This additional source of linear maps along which to pull back equations may allow for an ideal-theoretic version of the theorem. For details see [Sno13, DK13. 



\section{Bibliography}

[AH07] Matthias Aschenbrenner and Christopher J. Hillar. Finite generation of symmetric ideals. Trans. Am. Math. Soc., 359(11):5171-5192, 2007.

[AH08] Matthias Aschenbrenner and Christopher J. Hillar. An algorithm for finding symmetric Gröbner bases in infinite dimensional rings. 2008. Preprint available from http://arxiv.org/abs/0801.4439.

[AT03] S. Aoki and A. Takemura. Minimal basis for connected Markov chain over $3 \times 3 \times k$ contingency tables with fixed two dimensional marginals. Australian and New Zealand Journal of Statistics, 45:229-249, 2003.

[BB13] Weronika Buczyńska and Jarosław Buczyński. Secant varieties to high degree Veronese reembeddings, catalecticant matrices and smoothable Gorenstein schemes. J. Alg. Geom., 2013. To appear; preprint available from http://arxiv.org/abs/1012.3563.

[BD11] Andries E. Brouwer and Jan Draisma. Equivariant Gröbner bases and the two-factor model. Math. Comput., 80:1123-1133, 2011.

[Bor91] Armand Borel. Linear Algebraic Groups. Springer-Verlag, New York, 1991.

[CEF12] Thomas Church, Jordan S. Ellenberg, and Benson Farb. FI-modules: a new approach to stability for $S_{n}$-representations. 2012. Preprint, available from http://arxiv.org/abs/1204.4533.

[Coh67] Daniel E. Cohen. On the laws of a metabelian variety. J. Algebra, 5:267-273, 1967.

[Coh87] Daniel E. Cohen. Closure relations, Buchberger's algorithm, and polynomials in infinitely many variables. In Computation theory and logic, volume 270 of Lect. Notes Comput. Sci., pages 78-87, 1987.

[DEKL13] Jan Draisma, Rob H. Eggermont, Robert Krone, and Anton Leykin. Noetherianity for infinite-dimensional toric varieties. 2013. Preprint available from http://arxiv.org/abs/1306.0828.

[DK13] Jan Draisma and Jochen Kuttler. Bounded-rank tensors are defined in bounded degree. Duke Math. J., 2013. To appear; preprint available from http://arxiv.org/abs/1103.5336.

[dLST95] Jesús A. de Loera, Bernd Sturmfels, and Rekha R. Thomas. Gröbner bases and triangulations of the second hypersimplex. Combinatorica, 15:409-424, 1995.

[Dra10] Jan Draisma. Finiteness for the k-factor model and chirality varieties. Adv. Math., 223:243-256, 2010.

[DS98] Persi Diaconis and Bernd Sturmfels. Algebraic algorithms for sampling from conditional distributions. Ann. Stat., 26(1):363-397, 1998.

[DS06] Vesselin Drensky and Roberto La Scala. Gröbner bases of ideals invariant under endomorphisms. J. Symb. Comput., 41(7):835-846, 2006.

[DSS07] Mathias Drton, Bernd Sturmfels, and Seth Sullivant. Algebraic factor analysis: tetrads, pentads and beyond. Probab. Theory Relat. Fields, 138(3-4):463-493, 2007.

[DX10] Mathias Drton and Han Xiao. Finiteness of small factor analysis models. Annals of the Institute of Statistical Mathematics, 62(4):775-783, 2010.

[GSS05] Luis D. Garcia, Michael Stillman, and Bernd Sturmfels. Algebraic geometry of Bayesian networks. J. Symb. Comp., 39(3-4):331-355, 2005.

[GW09] Roe Goodman and Nolan R. Wallach. Symmetry, representations, and invariants, volume 255 of Graduate Texts in Mathematics. Springer, New York, 2009.

[Hig52] Graham Higman. Ordering by divisibility in abstract algebras. Proc. Lond. Math. Soc., III. Ser., 2:326-336, 1952. 
[HMdC13] Christopher J. Hillar and Abraham Martín del Campo. Finiteness theorems and algorithms for permutation invariant chains of Laurent lattice ideals. J. Symb. Comput., 50:314-334, 2013.

[HS12] Christopher J. Hillar and Seth Sullivant. Finite Gröbner bases in infinite dimensional polynomial rings and applications. Adv. Math., 221:1-25, 2012.

[Kru60] Joseph B. Kruskal. Well-quasi ordering, the tree theorem, and Vazsonyi's conjecture. Trans. Am. Math. Soc., 95:210-225, 1960.

[Lan65] Serge Lang. Algebra. Addison-Wesley Publishing Company, Inc., Reading, Mass., 1965.

[LM04] Joseph M. Landsberg and Laurent Manivel. On the ideals of secant varieties of Segre varieties. Found. Comput. Math., 4(4):397-422, 2004.

[LSL09] Roberto La Scala and Viktor Levandovskyy. Letterplace ideals and non-commutative Gröbner bases. J. Symb. Comp., 44(10):1374-1393, 2009.

[LW07] J. M. Landsberg and Jerzy Weyman. On tangential varieties of rational homogeneous varieties. J. Lond. Math. Soc.(2), 76(2):513-530, 2007.

[NW63] Crispin St.J.A. Nash-Williams. On well-quasi-ordering finite trees. Proc. Camb. Philos. Soc., 59:833-835, 1963.

[OR11] Luke Oeding and Claudiu Raicu. Tangential varieties of segre-veronese varieties. 2011. Preprint, avaibable from http://arxiv.org/abs/1111.6202.

[Rai12] Claudiu Raicu. Secant varieties of Segre-Veronese varieties. Algebra $\& 3$ Number Theory, 6(8):1817-1868, 2012.

[SHS07] Serkan Serkan Hoşten and Seth Sullivant. A finiteness theorem for Markov bases of hierarchical models. J. Comb. Theory, Ser. A, 114(2):311-321, 2007.

[Sno13] Andrew Snowden. Syzygies of Segre embeddings and $\Delta$-modules. Duke Math. J., 2013. To appear, preprint available from http://arxiv.org/abs/1006.5248.

[SS12a] Steven V. Sam and Andrew Snowden. GL-equivariant modules over polynomial rings in infinitely many variables. 2012. Preprint, available from http://arxiv.org/abs/1206.2233.

[SS12b] Steven V. Sam and Andrew Snowden. Introduction to twisted commutative algebras. 2012. Preprint, available from http://arxiv.org/abs/1209.5122. 